$\cos 2271-98$

\title{
HIGH-TEMPERATURE SERIES EXPANSIONS FOR ISOTROPIC HEISENBERG MODEL IN ONE AND TWO DIMENSIONS: t
}

\author{
P. D. Gujrott \\ Depertment of Physics \\ Columbio University \\ Now York, N.Y. 10027
}

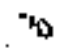
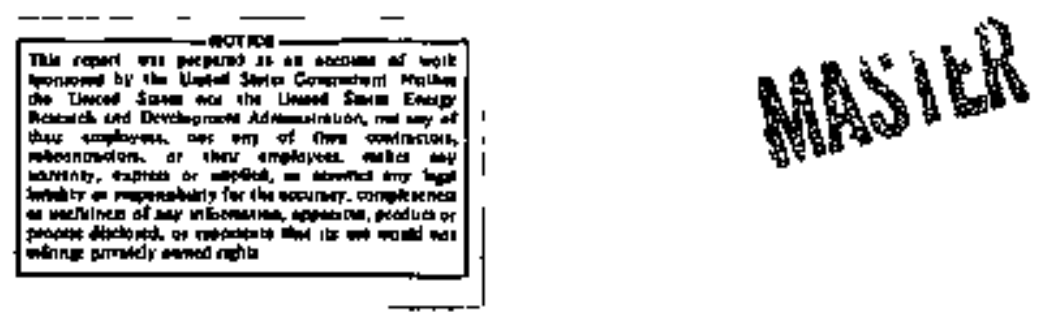

This reseorch wos supported in part by the U.S. Enetgy Resedreh and Development Administrotion 


\section{DISCLAIMER}

This report was prepared as an account of work sponsored by an agency of the United States Government. Neither the United States Government nor any agency Thereot, nor any of their employees, makes any warranty, express or implied, or assumes any legal liability or responsibility for the accuracy, completeness, or usefulness of any information, apparatus, product, or process disclosed, or represents that its use would not infringe privately owned rights. Reference herein to any specific commercial product, process, or service by trade name, trademark, manufacturer, or otherwise does not necessarily constitute or imply its endorsement, recommendation, or favoring by the United States Government or any agency thereof. The views and opinions of authors expressed herein do not necessarlly state or reflect those of the United States Government or any agency thereof. 


\section{DISCLAIMER}

Portions of this document may be illegible in electronic image products. Images are produced from the best available original document. 


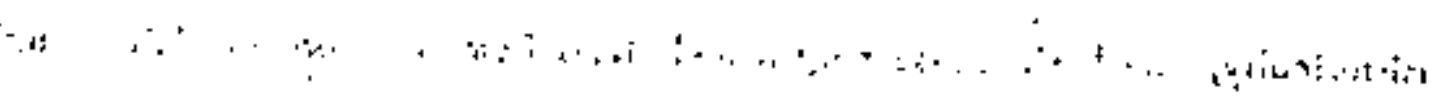

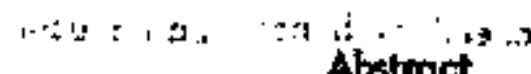

Abstroct

,e iasil- in. vist

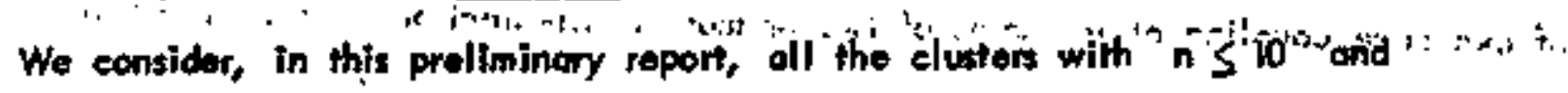

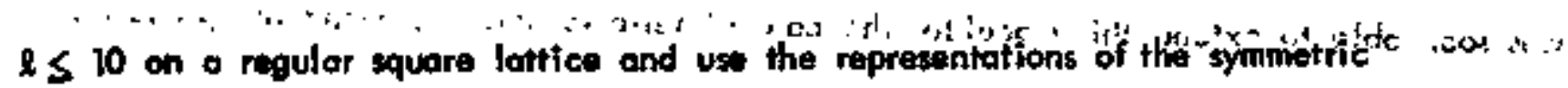

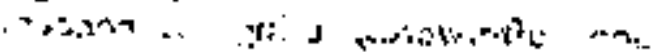

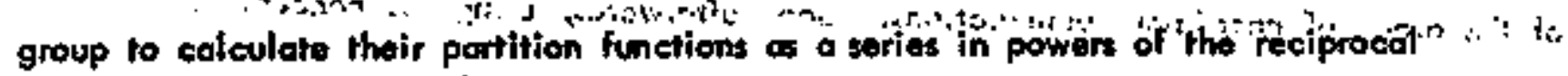

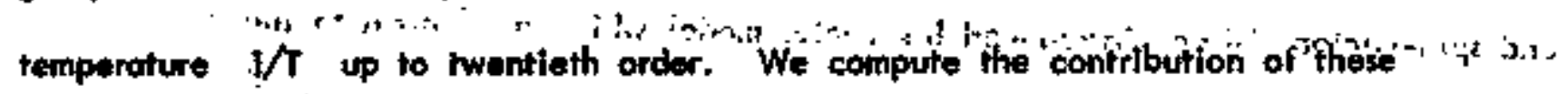

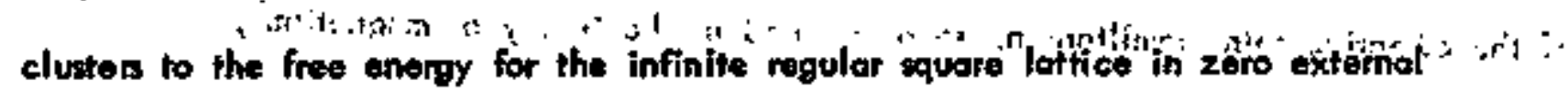
magnetic field. The contribution of the external field can be colculated exactly to any desired power in the reciprocol remperature. Wo have corried out this colculation for the linear, lottices.

The complote contribution to twentieth onder for on infinite square lottice must ineluce groph that oover remaining eluaters $4 \mathrm{p}$ to $n=20$ and $l=20$. The con-

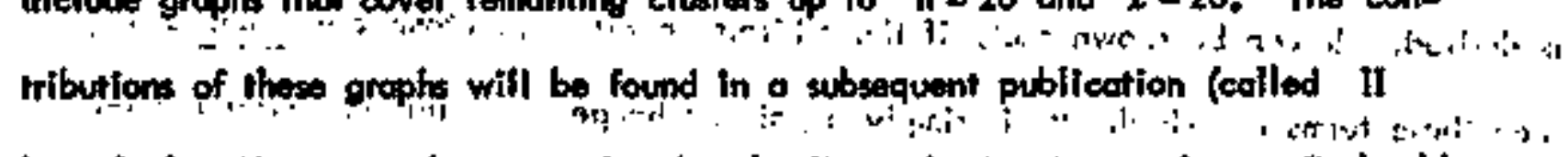
hereafter). Howaver, the expression tor the lineor lattice is complete. It should be mentioned that our compulation ore completely free of rounding errors.

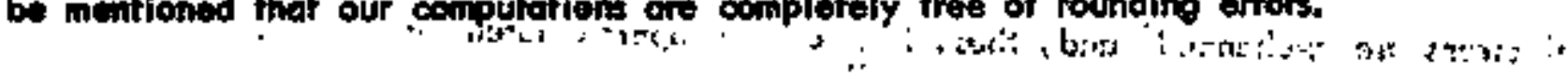

$$
\begin{aligned}
& \because \quad \therefore \quad \therefore \quad \therefore \%
\end{aligned}
$$

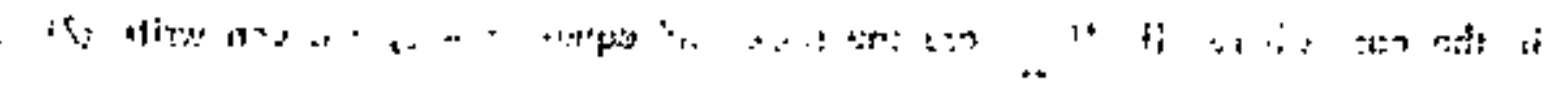


Section 1 Introduction:

Historicolly, the basic Heisenberg model stems from the work of Heirler and London', in which they proposed their theory of chemical bonds bosed upon the concept of exchange coupling of the spins of two or more multi-electron atoms. Meisenberg was soon oble to extend this model to the case of ferromognetiam, using the proporties of the group of coordinate permutations. Soon afterwards, using the concept of spin-space ond spin-vector, Dirac" introduced his vector model which gove bitth to the concept of the effective spin Hamiltonion, so often used in the theory of mognetisn 4 ,

$$
H_{\text {eff }}=\underset{\text { (P) }}{\Sigma} H_{P} P
$$

acting in the isomorphic spinspoce, the sum boing taken over alf possible N! permulations of the $N$ electrons involved. Here $H_{p}$ ineludes the pace coordinate integration of the original Hamiltonian for the system whose effective spin Hamiltónion is desited. It can be shown that, if the ofbitals on different atoms are almost orthogonal, then those terms for which $P$ is simply a pair exchange ' $P_{i j}$, interchanging the $i$-th and j-th electron spin labels, are much lorger than those for which more than two electrons are exchanged ${ }^{4}$ and, thus, $\mathrm{H}_{\text {eff }}$ can be approximatied by

$$
H_{\text {eff }}=\underset{\text { (poir) }}{\Sigma} H_{P_{i j}} P_{i j} ;
$$

in the case where all $H_{P_{i j}}$ ane the same and equal to $-J$, we can write (2) os

$$
H=H_{\text {eff }}=-J \underset{\text { (j) }}{\Sigma} P_{\text {ij }}
$$


It was Dirae who observed that the permutation operotor $P_{i j}$ which permutes the rwo spin variables $i$ and $j$ in the case of electrons with $s=1 / 2$ con be written in terms of the Pauli matrices;

$$
\begin{aligned}
P_{i j} & =\frac{1}{2}\left(i+\vec{\sigma}_{i} \vec{\sigma}_{j}\right) \\
& =\frac{1}{2}+2 \vec{s}_{j} \cdot \vec{s}_{j}
\end{aligned}
$$

whore $\vec{a}$ are the Pouli motrices and $\vec{s}$ are electron spin operotors. Thus, we observe thet $H$ in (B) is indeed equivalent to the bosic Heisenberg model apart from a constant tem 1/2, which does not alter the thermodynamics of the system.

In the sum that appears in $(3)$, the suffices $i$ and $j$ refer to the electron spin variobles $i$ and $j$, the electrons themselves being locoted ot the $i$-lh and $j$-th lattice points of the lattice $L$ (fintte or infinite) and (ij) denotes a pair of lattice poin's in Li each such pair is counted only once in $H . J$ is called the exchange energy. From now on, we will restrict ourselves only to the nearest neighbor pairs (j) in the sum in (3).

We will not paute here to assess the theoretical background to (3) but refer the interested reoder to the articles by Anderson 5 and Steveris ${ }^{6}$. Throughout this Paper, we traat (3) merely as a mathematical model Haniltonion which con easily be generolized to any higher spin s. $P_{i j}$ is still a permutation operator permuting the spin variablet $i$ and $j$ but now we do not have any simple form for $P_{i j}$ like (4) which is good for $s=1 / 2$. Although we do not make use of (4) for $P_{i j}$ with $s=1 / 2$, we stifl restrict ourselves to that cose because the method to be described below is most successfut with $s=1 / 2$ and also becouse we con compare our rosult 
with known results for the spin $-1 / 2$ rotrople Heisenberg model. We are aloo going to restrict ounselves to the regular square lattice throughout this work and study the obove model (3) by the establiahed method of high-temperature exponsion, known as the finite cluster method, in terms of the expansion parameler $\beta=1 / k_{B} T$ up to 20-th power.

We will sketch briefly the historical background of the high-temperature series expansions for spin - 1/2 isotropic Heisenberg model. Most of the works have been done in three dimensions and, os such, can not be useful for comparison with our results. The first colculation was carrifad out by Opechowski ${ }^{7}$ who used a method first proposed by Kramer. He reported the first four terms of the expansion for f, c. c. lattice. Zehler" carried out the calculation for s,c. lattice. Rushbrooke and Wood extended the colculation to include the fifth order tem. Domb and Sykes 10 added the sixth rem. With the use of the fintre cluster method, Domb and Wood 11 extended the calculation to include the seventh and aighth order tems for open lattices. Wood ${ }^{12}$ and Demb and Wood ${ }^{13}$ calculated the next, higher termis for open lattices.

The Hamitanian (3) for $s=1 / 2$ was used, as far as the high-temperature serie expansion is concerned, for the first time by Domb and 5 kes ${ }^{14}$. They used this Mamiltonian to check the calculations of Aushbrooke and Wood 90 by using the foct that $P_{i j}$ belong to the symmetric group. If is precisely this property of $P_{i j}$ that endbled Baker and co-warkers ${ }^{15}$ to colculate the first 21 terms for a linear Heisenberg choin. They had used the irreducible repressentations of the symmetric group to calculate the various troces involved in the partition function. Later, they gave the first nine terms for f.c.c. lattice and the first ten terms for b. c. c, and s. c. lattices. They corried out similar work on planor lattices and gave the fiest ten tarms for triangylar 
and square lottices. In one sense, they carried the previous work further by colculating the effect of the external mognefic field to the elghth order in the magnetic field and in the tnverse tempereture.

Using a similar approach, to be discussed it detail in the following section, we hove been able to extend the work of Baker and co-morkers 150,150 . We report here the contributions of all fintte clusters, open and closed, ratisfying $n \leq 10$ and $l \leq 10^{*}$, to the free energy for the infinite square lattice up to twentieth power in $\left(\mathrm{J} / 2 \mathrm{k}_{B} T\right)$. Wo have taken the extemal mognetic field to be cbsent. .However, wo hove corried out the colculation in the presence of the field in the cose of the linear chain, and report the result, which is good to all onders in the mognefic fleld but good only up to tifh order in the inverse remperature.

The plan of the peper will be as follows. We report in Section 2 the main polnts of the finite cluster method necessery for our penpose. It is this method that has been used offectively to coleulate higher tems in previous wok. The mothod hos been restricted to the $s=1 / 2$ case, for which it hos afroody produced the longest series yet publishad, because of the computational advantages. We discuss in Section 3 the we of the representations of the symmetric group to calculate the various traces of the Homilmonion (B). Section 4 deals with the actual computation of these traces. In Section 5, we inelude the mognetle field and discuss how its effect con be computed exactly to all orders in the field. Section 6 explains various steps involved in actual computation of the free energy and how they are occomplished. The various chedking

\footnotetext{
The tinal draft of the paper hawever, will include contributions of all finite elusters with ins 10 .
} 
$-6-$

procedures to convince us of the accuracy of our computational procedure are explained in the following section and the final section contains certain remarks about the advantages and shortcomings of our procedure. The result that we present for the regular square lattice is incomplete in that it does not include the contributions of graphs that cover clusters $\ell \geq 10$, but $n \leq 20$. However, the result is complete up to the eleventh power in $J / 2 k_{B} T$. The results for the linear chain are complete to the given order in $\mathrm{J} / 2 \mathrm{k}_{\mathrm{B}} \mathrm{T}$. The complete result up to 20 th order for the regular square lattice will be reported in a subsequent publication (At) : 
Section 2: The Finite Cluster Method

Now we turn our attention to the finite cluster method for high-temperature expensions, first proposed by Domb ${ }^{16}$. The method has been later olaborated by seyeral other authors ${ }^{17-20}$. We refer the reader. to a complete and readuble exposition of the merhod by Rushbrooke, Boker and Wood ${ }^{21}$ for further detoils. It should, however, be pointed out that there are of fow differences in our definitions and notations.

For definiteness, lef us consider a regular squore lattice $L$ of $N$ regularly spated lotticed points and $M$ nearest-neighbor pair-interactions $\dot{P}_{i j}$ defined over the $N$ points: $L=\{N, M\}$. For convenience, we will use pertiodfe boundary conditions. The method, however, holds for any kind of lattice. Let us imagine a set of $n$ " points and $\&$ pairinteroctions of $L$ such that each pair-intemation $P_{i j} \in\{l\}$ defines the interaction batween the two points $\left.I_{,}\right\} \in\{n\}$. If, moreover, the set $\{n, l\}$ is connected, we call the collection $\{n, l\} \quad$ cluster and denote it by $l_{n, l}, \tau$ where $\tau$ is a subscript which differentioke between many elusters with the some volues of $n$ and 2 . It is not necissary thot the cluster $L_{n, \ell, T}$ contain all the pir-intercctions originally between the $n$ lottice points on L. An example of o eluster with 6 lottice points and 6 pairinteractions is shown in Fig. 1 where the interaction $P_{2,5}$, originally in $L$, is obsent. If, however, we include $P_{2,5}$, we get another cluster with the same set of points, but with 7 poirminteractions.

The method to be deseribed below applies equally well whether we deal with $L$ or $L_{n, l, \tau}$. We witl use $L$ to denote either $L$ or $L_{n, l, \tau}$, if there is no confusion. The partition function for the Hamiltonion (1.3) defined over $L$ is given by

\#W6 oro going to take the extemal magnetic field to be chsent in this section. However, the melthod is valid oven in the presence of the-field. 


$$
\begin{aligned}
& -8- \\
Z_{L}(H) & =\operatorname{Tr} \exp \left(-H / k_{B} T\right) \\
& =\operatorname{Tr} \exp \left(\beta \underset{(i j)}{\sum} P_{i j}\right)
\end{aligned}
$$

where $\beta=J / k_{B} T$, the expansion parameter and the troce refers to the matrix representations of the aperatort $P_{i j}$. in the $2^{N}$-space in the case of $s=1 / 2$. After expanding, we get

$$
Z_{L}(H)=2^{N}\left[1+\operatorname{Tr} \underset{m=1}{\sum} \frac{\beta^{m}}{m !} \frac{1}{2 N}\left(\underset{(i j)}{\sum} P_{1 j}\right)^{m}\right] .
$$

Here, we have taken out the factor $2^{N}$ corresponding to $m=0$ in the sum so that the first term inside the square brocket, which' is indopendent of $\beta$, is unity. We will Find it convenient to define the reduced portition function $\mathrm{Z}_{\mathrm{L}}(\mathrm{H})$, such that

$$
Z_{L}^{\prime}(H)=Z_{L}(H) / 2 N
$$

Then,

$$
Z_{L}^{\prime}(H)=1+\operatorname{Tr} \stackrel{\infty}{m=1}_{m}^{\infty} \frac{\beta^{m}}{m !} \frac{1}{2^{N}}\left(\sum P_{i j}\right)^{m}
$$

To each ordered product of the pair-interactions $\mathrm{P}_{\mathrm{ij}}$ in the expansion of $\left(2 \mathrm{P}_{\mathrm{ij}}\right)^{\mathrm{m}}$ can be associoted a localized Ibelled groph localized on $L$ in which each bond between the points $i$ and $j$ of the groph corresponds to one of the $P_{i j}$ in the ordered product. The bonds in the graph are numbered according to the positions of the corresponding $P_{i j}$ in the ordered product from, say, left to right. Thus, the possible three ferms $P_{1,2} P_{1,2} P_{2,3}, P_{1,2} P_{2,3} P_{1,2}$ and $P_{2,3} P_{1,2} P_{1,2}$ that occur in the exponsion of $\left(2 \mathrm{P}_{\mathrm{ij}}\right)^{3}$ give rive to the three localized labelled graphs on $L$ as shown in fig. 2. To these three localized labelled grophs corresponds a single lacalized unlabelled graph, shown in Fig.3, where the latbelling on the bonds have been suppressect only the points hove their positions on 1 designated. We can 
simillarly define an unlocelized labelled groph which does not hove the points designated on $\mathrm{L}$, but has the bonds labelted. We can, suppres the labelling of the bonds and the designation of the points in the graph, and eall such graph a free graph. We will denote a free groph by $G_{n, m, t}$, the corresponding localized unlabelled graphs by. $G^{\prime}, m, t^{\prime}$, the unlocalized labelled graphs by $\bar{G}_{n, m, \bar{p}}$ and the localized labelled grapha by. $\vec{G}_{n, m, \bar{r} \cdot}$.

It is cleor thot the number of various $G^{\prime}, m, t^{*}$ corresponding to any given $G_{n, m, t}$ depends only upon the geometry of the lattice or the cluater and, hence, is colled the lottice constiont or the cluster constant as the case moy be.c. It wlll be denoted by $\lambda\left(G_{n, m,}\right)$. The number of $\bar{E}_{n ; m, t}$ corrosponoting to any $G_{n, m, t}$ depends upon $m$ and the multiplietities of the multiple bonds of $G_{n, m, t}$ and, hence, its called the multiplticity constant. 'If will be denoted by $v\left(G_{n, m}, i\right)$. If $G_{n, m, t}$ hos \& multiple bonds with multiplicity $m_{l}, m_{2}, \ldots, m_{\ell}$ such that

$$
\sum_{i=1}^{2} m_{i}=m
$$

then

$$
v\left(G_{n, m, t}\right)=\frac{m !}{\prod_{l=l}^{l} !}
$$

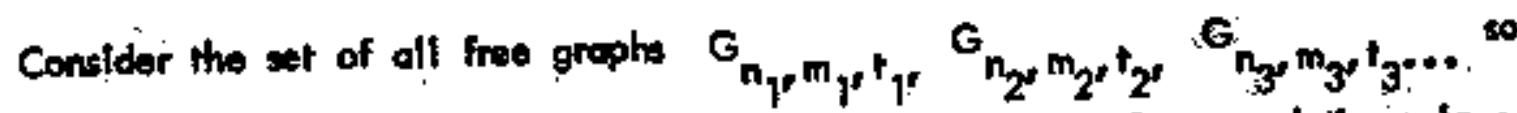
that, whon overy inultiple thend between ony two. points of any free graph is replaced by a

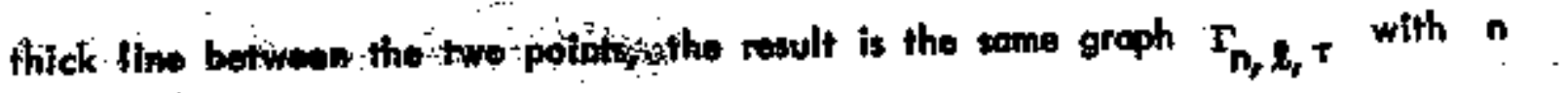


points and $\ell$ thick lines. The new graph is called the skeleton graph or the basic graph and represents a sel of free graphs obtained by replacing each thick line by multiple bonds of any multiplieity including 1. Of course, we do not consider bonds with zero multiplicily. The set of all graphs with fixed $m$ that correspond to $\Gamma_{n, L, T}$ will be denoted by $\Gamma_{n, l_{t} T}^{(m)}$. If $S\left(G_{n, m, l}\right)$ is the symmetry number of any free groph and if $G_{n, l, t}$ is a free groph similar to $\Gamma_{n, l, \tau}$ except thot each thick line of $\Gamma$ is replaced by a single bond, then with the following definitions:

$$
\begin{aligned}
& S\left(\Gamma_{n, l, \tau}\right)=S\left(G_{n, l, \ell}\right), \\
& \lambda\left(r_{n, l, T}\right)=\lambda\left(G_{n, l, t}\right) .
\end{aligned}
$$

it is exsy to see that

$$
\lambda\left(G_{n, m, t}\right)=\frac{S\left(\Gamma_{n, l, \tau}\right)}{S\left(G_{n, m, l}\right)} \lambda\left(\Gamma_{n, \ell, \tau}\right) .
$$

After this brief digression about the graph terminolagy, we turn our attention to (4). A typical groph $\bar{G}_{n, m,}^{\prime}$, in the expansion involves only $n$ out of $N$ points of $\mathrm{L}$, and when we take the trace, the remaining points contribute a factor $2^{\mathrm{N} w n}$, leoving a foctor $2^{\mathrm{n}}$ in the denominator. As this foctor appears in the trace of all grophs corresponding to $G^{\prime} m_{,} m_{t}{ }^{\prime \prime}$ we define the mean trace $T\left(G_{n, m, t^{\prime}}\right)$ as

$$
T\left(G_{n, m, l}\right)=T\left(G_{n, m, t^{\prime}}^{\prime}\right)=\frac{T}{2^{n} v\left(G_{n, m, p}\right)} \sum_{G_{n, m, t^{\prime}}} \operatorname{Tr} \prod_{(j)} P_{i j} .
$$

In the presence of on external magnetic field, 2 is replaced by $\zeta$ (see Section 5), and $T$ (G) becomes a polynomial in $\mu$ :

$$
\left.T\left(G_{n, m, t}\right)=\frac{1}{\zeta^{n} v(G)} \sum_{\left(G^{\prime}\right)} \operatorname{Tr}\left(\pi P_{i j}\right) \exp \left(2 \gamma S_{3}\right)\right)
$$


where the sum is over all $\bar{G}_{n, m,}^{\prime} \bar{r}^{\prime}$ corresponding to $G_{n, m, r^{\prime}}^{\prime}$. We have used the obvious fact that $T\left(G_{n,}^{\prime}, f^{\prime}\right)$ is independent of its localization on $L$ and, therefore, write it $T\left(G_{n, m}, r\right)$. With the help of $(B),(A)$ com be expressed as foltows:

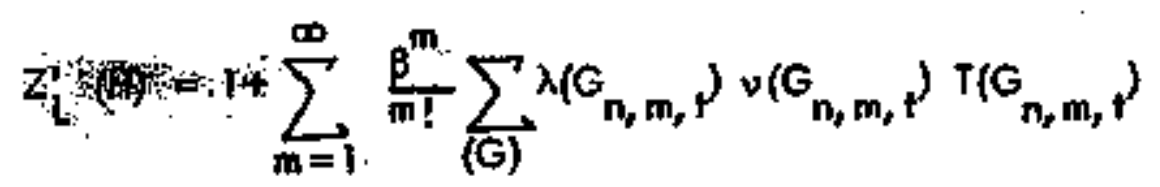

and the sum over (G) means the sum over all possible free graphs $G_{n, m, t}$. We now define the cumulant or the Uhsell function $U\left(G_{n, m}, j\right.$ from the moments ar the meon iraces $T\left(G_{n ; m, t}\right)$ in the usual way:

$$
\tau\left(G_{n, m, t}\right)=\sum_{k \text { P }} \sum_{(m, k)} \prod_{i=1}^{k} U\left(G_{n_{i}, m_{i}, t}\right)
$$

such that $2 m_{i} \cdots m$. Hero $P(m, k)$ denotes tho partition of $m$ bonds of $G, n, m, t$ into $k$ partsiand any such portition defines uniquely $k$ overtapping graphs $G_{n_{i}}, m_{i}, t_{i}$ For cointing pumpones, we asame thet all bonds in $G_{n, \dot{m}, t}$ are labelled and find all possible distinct portitiens $P(m, k)$ of these in bonds into $k$ ports. The importonce of the Urisoll finction $\mathbf{U}$ is due to the fact that it vanishos for disconnected groph. Using the Uraell function, we con write down the expression for en $Z_{L}^{\prime}(\mathcal{H})$ in a form similar to $(9)$ :

$$
\ln Z^{\prime} L^{(H)}=\sum_{m=1}^{\infty} \frac{\beta^{m}}{m^{m}} \sum_{(G)} \lambda\left(G_{n, m, t}\right) v\left(G_{n, m, l}\right) U\left(G_{n, m, t}\right)
$$

and the sum over (G) is now only over all connected free graphs. Using (7), we con write (11) os follows:

$$
\text { ln } Z^{\prime}{ }^{(H)}=\sum_{\Gamma_{n, l, \tau}} \lambda\left(\Gamma_{n, l, \tau}\right) \vee\left(\Gamma_{n, l, \tau}\right)
$$


where the sum is over all skeleton grophs, and $V$ is given by

$$
\begin{aligned}
& V\left(\Gamma_{n, \ell, \tau}\right)=\sum_{m=\ell}^{\infty} \frac{\beta^{m}}{m !} \sum_{(G)}^{\Gamma_{n !}^{(m)}, T} v\left(G_{n, m, t}\right) \frac{S\left(\Gamma_{n, \ell, T}\right)}{S\left(G_{n, m, l}\right)} U\left(G_{n, m, l}\right) \\
& =\sum_{m=\ell}^{\infty} \frac{\beta^{m}}{m !} v_{m}\left(\Gamma_{n, l, q}\right) ;
\end{aligned}
$$

$$
v_{m}\left(\Gamma_{n, \ell, \tau}\right)=\sum_{(G)}^{\Gamma_{n_{2}, \ell, T}^{(m)}} v\left(G_{n, m, t}\right) \frac{S\left(\Gamma_{n, \ell, T}\right)}{S\left(G_{n, m, l}\right)} U\left(G_{n, m, t}\right)
$$

The sum over (G) in (13) and (14) is over all free graphs of given th that correspond to the same skeleton graph $\Gamma_{n, 2, r}$. The sum over $m$ starts from $t$ becouse fninimum $m$ for this set (G) is $\ell$. As $L_{n, \ell_{t} \tau}$ and $\Gamma_{n, \ell_{t},}$ ore topologically identical except that the thick line in $T_{n, l, T}$ is replaced by the pair-interaction $P_{i j}$ in $L_{n, \ell, T}$, we can replace $\Gamma_{n, \ell, T}$ in (12), (13) and (14) by $L_{n, \ell, T}$. Thus,

$$
\ln Z_{L}^{E}(H)=\sum_{L, L, T} \lambda\left(L_{n, \ell, T}\right) V\left(L_{n, \ell, \tau}\right)
$$

17 is clear that $V\left(L_{n, l, T}\right)$ depends only on the comected graphs $G_{n, l, t}$ that cover the whole cluster $L_{n, L_{r}} T$ and the structure of the cluster $L_{n, \ell_{r}}$, with respect to $L$ enkers through the lattice constont $\left.\dot{\lambda}_{\left(\mathrm{n}_{\ell, \ell}, \mathrm{T}\right.}\right)$ which gives the embedding of the cluster in $L$. Thus, $\ln Z^{\prime} L^{(H)}$ involves two independent quantities $\lambda$ and $V$. that can be colculated separately. 
If we have, instead of the infinite lattice $L$, the cluster $L_{n, L_{,}}$, then (15) con be written as

$$
\ln Z_{L^{\prime}, L^{\prime}, \tau}^{(H)}=\sum_{L_{n^{\prime}, \ell^{\prime}, T^{\prime}}} \lambda\left(L_{n^{\prime}, \ell^{\prime}, \tau^{\prime}}\right) \vee\left(L_{n^{\prime}, \ell^{\prime}, T^{\prime}}\right)
$$

where $\lambda\left(L_{n^{\prime}}, \ell^{\prime}, q^{\prime}\right)$ is the number of ways to embed $L_{n^{\prime}, \ell^{\prime}, \tau^{\prime}}$ in $L_{n, l, \tau^{\prime}}$, that is, tho cluster constant. If we are interested in en $Z_{L}$ (N) up to given order $m$ in $\beta$, we need consider only those finfte elusters thet hove at most $m$ pair-interactions defined over them. This is obvious from the fact that the sum over in in (13) starts from $\&$. Wo compule $\nabla\left(R_{n, l, q}\right)$ for these elusters by inverting the celation (16), or by successive elimination. By pulting the Defonction of each finite clister in (15) we obfain te $Z_{\mathrm{L}}^{\prime}(\mathrm{H})$, the truneated free energy up to m-th power in $\beta$. Thus, we nead the $\vec{V}$ functions of all finite clusters with $2 \leq m$ pair-interactions: It is for this reoson thet the present method is known an the Frilte cluater method.

Let us illustrate the meaning of (16) by considering finst. fow clusters on the square lattice $L$, shown in Fig.4. The only sub-cfuster for $L_{1}$ is itself. Therefore,

$$
\ln Z_{L_{1}}^{\prime}(H)=V\left(L_{1}\right) \text {. }
$$

From $L_{2}$ con be formed two different sub-elusters $L_{1}$, and, then, there is $L_{2}$ itsels. Thus,

$$
\ln Z^{\prime} L_{2}(H)=V\left(L_{2}\right)+2 V\left(L_{1}\right)
$$

*From now on, the bar over a polynomial in 8 will meon its inuncoled form up to the desired power in . In that cose, we should reploce in $Z^{\prime}$ and $V$ in (15) and (16) by their truncoted forms, viz. $\overline{l n} \bar{z}$ and $\nabla$. 
For $L_{3}$, there are three.different subuclusters $L_{1}$, two $L_{2}$ and one $L_{3}$;

$$
\ln \mathrm{Z}_{\mathrm{L}_{3}}^{\prime}(\mathrm{H})=\mathrm{V}\left(\mathrm{L}_{3}\right)+2 \mathrm{~V}\left(\mathrm{~L}_{2}\right)+3 \mathrm{~V}\left(\mathrm{~L}_{1}\right)
$$

In this way, the remaining relations (16) con be written down for the rest of the elusters:

$$
\begin{aligned}
& \ln Z_{L_{4}}^{\prime}(H)=V\left(L_{4}\right)+3 V\left(L_{2}\right)+3 V\left(L_{1}\right) \\
& \ln Z_{L_{5}}^{\prime}(H)=V\left(L_{5}\right)+2 V\left(L_{3}\right)+3 V\left(L_{2}\right)+4 V\left(L_{1}\right) \\
& \ln Z_{L_{6}}^{\prime}(H)=V\left(L_{6}\right)+V\left(L_{4}\right)+2 V\left(L_{3}\right)+4 V\left(L_{2}\right)+4 V\left(L_{1}\right) \\
& \cdot \ln Z_{L_{7}}^{\prime}(H)=V\left(Q_{7}\right)+4 V\left(L_{4}\right)+6 V\left(L_{2}\right)+4 V\left(L_{1}\right)
\end{aligned}
$$

from these relations, $V\left(L_{1}\right), v\left(L_{2}\right) \ldots$ can be calculated by successive eliminations." Once V-functions for all clusters has been computed, we detemine in $Z_{1}^{\prime}(1)$ for the infintre lattice by multiplying eoch $V\left(L_{n, l, r}\right)$ by the corresponding $\lambda\left(L_{n, l, \tau}\right)$, and using (15) ..

Instead of computing $T\left(G_{n, m, t}\right)$ for all free graphs that occur in the exponsion of $\overline{Z_{L}^{\prime}}(H)$, it is convenient to compute the sum of all $T\left(G_{n, m, t}\right)$ for a given $m$ by computing the troce of the muth power of the Haniltonian for all finite clusters as long os $n$ is small. We have done this for all finite elusters with $n \leq 10$ by the method discussed in the next two sectlons. Once $\vec{Z}^{\prime}$ for each cluster is computed, We could inctude in the sum in (15) the tem $V\left(L_{1}, 0\right)$ corresponding to $n=1, q=0, i, \theta$, , a single point and put $V(L, 0)=\ln 2$. In this cose, the right hand side of (15) becomes 
$\overline{\ln } \overline{Z^{\prime}}$ can be computed easily and then by using relations itke (17), various $\nabla\left(L_{n, l, \tau}\right)$ can be computed. It is this part of the computation that is presented in this paper. The cornputotion of $t$ and $U$ for graphs covering more than ten lattice poink is done by another method, which will be the subject matter of the sacond paper. of the present series.

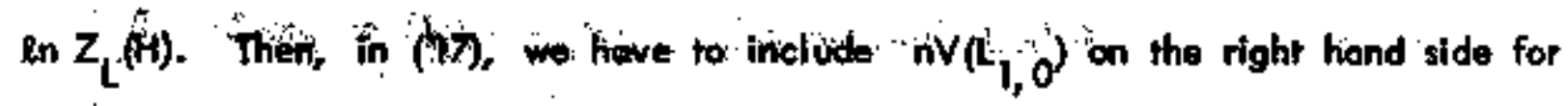
each eluster. To ovoid including this rerm, we aro going to deal with $\ln Z_{L}^{\prime}(H)$, instood of $\ln \mathrm{Z}_{\mathrm{L}}(\mathrm{H})$. 
Section 3. Representations of the Symmetric Group

The reoson that we used $P_{i j}$, a tromsposition optrator, as the form of the pair-intergation in our Homiltonian rather than the usual $\vec{s}_{i} \vec{s}_{j}$ is that the interaction term $P_{i j}$ and any product of a number of them can be. treated as. a permutation on the spin indices involved. We observe that ony product of $P,{ }^{\prime}, \ldots$ defined over a cfuster $L_{n, l,}$, belong to the symmetric group $\$(n)$. This property enables us to use the stondard theory of representations of the symmetric group ${ }^{22}$ to find the partition function, which involves raking the traces of the various powers of H. For $s=1 / 2$, the spin-space for the cluster $L, l, r$ hos the dimention $2^{n}$ and the different powers of $H$ that appear in $Z_{L} \quad(H)$ are also of the order $2^{n}$ in a spin-space in which we can choose the following $2^{n}$ base-vectars:

$$
\phi(1) \phi(2) \phi(3) \ldots \ldots \ldots \phi(n-1) \phi(n)
$$

where $(1)$ represents o spto-up state a(t) or a spin-down stote $\beta(i)$. Replocing each $a(1)$ by 1 and $\beta(i)$ by $x_{i}$ oppearing in the set (I) of the base-vectors, we can arrange the $2^{n}$ states according to the following scheme:

$$
\begin{aligned}
& x_{1}, x_{2}, \ldots x_{n} \\
& x_{1} x_{2}, x_{1} x_{3}, \ldots, x_{n-1} x_{n}
\end{aligned}
$$

$$
x_{1} x_{2} x_{3} \ldots x_{n}
$$


such that there are precisely $\left(\begin{array}{l}n \\ k\end{array}\right)=\frac{n !}{k !(n-k) !}$ states in the $k-1$ h row, $k=0,1,2, \ldots, n_{+}$ These states account for all $2^{n}$ states (l). All states, belonging to any row hove the same value of the 3-component of the tolat spin $\vec{s}$ :

$$
s_{3}=\frac{1}{2}(n-2 k) \quad k=0,1,2, \ldots n
$$

Thus, the vorious subuspoces $\mathrm{R}^{(k)}$ spanned by the bast-vectors in the $k$-ith row correspond to a fixed value of $S_{3}$ given by $(3)$. It is easily seen that each of these subn spaces $R^{(k)}$ remains invoriant under any $P_{i j}$ or any product of $P_{i j} s$ and, therefore, under $S(n)$. Thus, the original $2^{n}$-order matrix representation of $P_{i j}$ or $H$ can be broken down into $(n+1)$ different motrix representations of order $\left(\begin{array}{l}n \\ k\end{array}\right), k=0,1, \ldots, n$. We denote these representations by $\mathrm{P}_{\mathrm{ij}}{ }^{(k)}$ or $\mathrm{H}^{(\mathbf{k})}$.

Let us construct the matrix representations $p_{i j}^{(k)}$ and $H^{(k)}$. We choose the bose vectors of the k-th row in (2) os the besis of representations in the subuspace $R^{(k)}$. Since each basenvector with given volue of $S_{3}$ is tronsformed by $P_{i j}$ into another with the same value of $S_{3}$, there is only one element in each row of the matrix representation $\mathrm{P}_{\mathrm{ij}}^{(k)}$ which is unity and the rest of the elements of the row are zero. Since there are l. pair-inferaction in $H$, the matrix representolion $H^{(k)}$ will have precisely at the most 2 non-zero elements in each row. The matrix representation $H^{(k)}$ is sparse. We note that $H^{(k)}$ is a symmetric matrix. It is also a positive makrix and, therefore, the fraces of all powers of $H$ are positive. As $H^{(k)}$ and $H^{\text {(n- :.: }}$ are equivalent represtentations, we need only find the representations $H^{(0)}, H^{(1)}, H^{(2)} \ldots, H^{([n / 2])^{*}}$ and write the malrix repiesentertion $H$ for the Hamiltanian as

$$
H=H^{(0)} \otimes H^{(1)} \otimes H^{(2)} \otimes \cdots \otimes H^{(n-1)} \otimes H^{(n)}
$$


The order $\left(\begin{array}{l}n \\ k\end{array}\right)$ of the motrix representotion, $H^{(k)}$ rapidly increases with $n$. For example, the orders of the representations corresponding to $k=0,3,2,3,4$ and 5 for $n=10$ are

$$
1,10,45,120,210 \text {, and } 242
$$

while the corresponding orders for $n=11$ are

$$
1,11,55,165,333 \text { and } 462 \text {. }
$$

The largest order for $n=11$ is almost twice asilange os that for $n=10$. Therefore, the present method, to be described in defail in the next two sections, becones. impracticable for $n>10$. We have used the present method to calculate the partition functions for all the elusters with $n \leq 10$, except in the case of one dimension where we have also colculated the partition function for the II-point lineor eluster. The contributions of the rest of the graphs covering more than ten latrice points are colculated by means of o different technique which will be reported in detait in a subsequent publication (II). 
Section 4 . Computation of $\operatorname{Tr}\left(H^{(k)}\right)^{m}$

We will discuss below the octual compintation of the traces of the various powers of the Hamiltontan in the invariont sub-spaces $R^{(k)}$ for any cluster $L n, 2, \tau$. It should be emphosized that throughout our computation, we deal only with infegers, thereby making the etror in our results absolutely zero. In this sense, our results are exack through the order in $p$. Fhat wo ore interested in.

Let us represent the typical matrix element of $H^{(k)}$ by $h_{i j}$. We find it convenient to write the secular oquation for this motrix as

$$
\operatorname{det}\left|\mu^{h_{1 j}}-1\right|=0
$$

which differs from the usual equation det $\left|h_{i j}-\lambda\right|=0$ in that we have replaced $\lambda$ by $1 / \mu$. The determinant in (i) is a polynomical of order $\left(\begin{array}{l}n \\ k\end{array}\right)$ in $\mu$ :

$$
p^{(k)} \cdot(\mu)=P_{0}^{(k)}+P_{1}^{(k)} \mu+P_{2}^{(k)} \mu^{2}+\ldots+P_{k}^{(k)} \mu^{k}=0
$$

where $k=\left(\begin{array}{l}\mathrm{D} \\ k\end{array}\right)$. The term $\mathrm{P}_{0}^{(k)}$ is either +1 or -1 , depending upon whether $k$ is even or odd. If $\lambda_{1}, \lambda_{2}, \ldots, \lambda_{\kappa}$ are the eigenvalues of the Hamitronian in the sub-spoce $R^{(k)}$, then we note that

$$
\begin{aligned}
& \Sigma \lambda_{i}=p_{1}^{(k)} / p_{0}^{(k)} \\
& \sum \lambda_{i} \lambda_{j}=g^{(k)} / p_{0}^{(k)} \\
& \because \neq j \\
& \sum \lambda_{j} \lambda_{j} \lambda_{k}=p_{3}^{(k)} / p_{0}^{(k)}
\end{aligned}
$$


and it is exy to ftind vortous $\sum_{i=1}^{k} \lambda_{i}^{m}$ for different $m$ 's from these equations. Since we ore interested in finding $\sum_{i=1}^{k} \lambda_{i}^{m}$ for $m=1,2, \ldots, 20$, we can fruncote the polynomied $\mathrm{p}^{(k)}(\mu)$ in $(2)$ to the first twenty-one remsi"

$$
\beta^{(k)}(\mu)=p_{0}^{(k)}+P_{1}^{(k)} \mu+\ldots p_{20}^{(k)} \mu^{20}
$$

Let us write out the seculor deteminont in (1) explicitly :

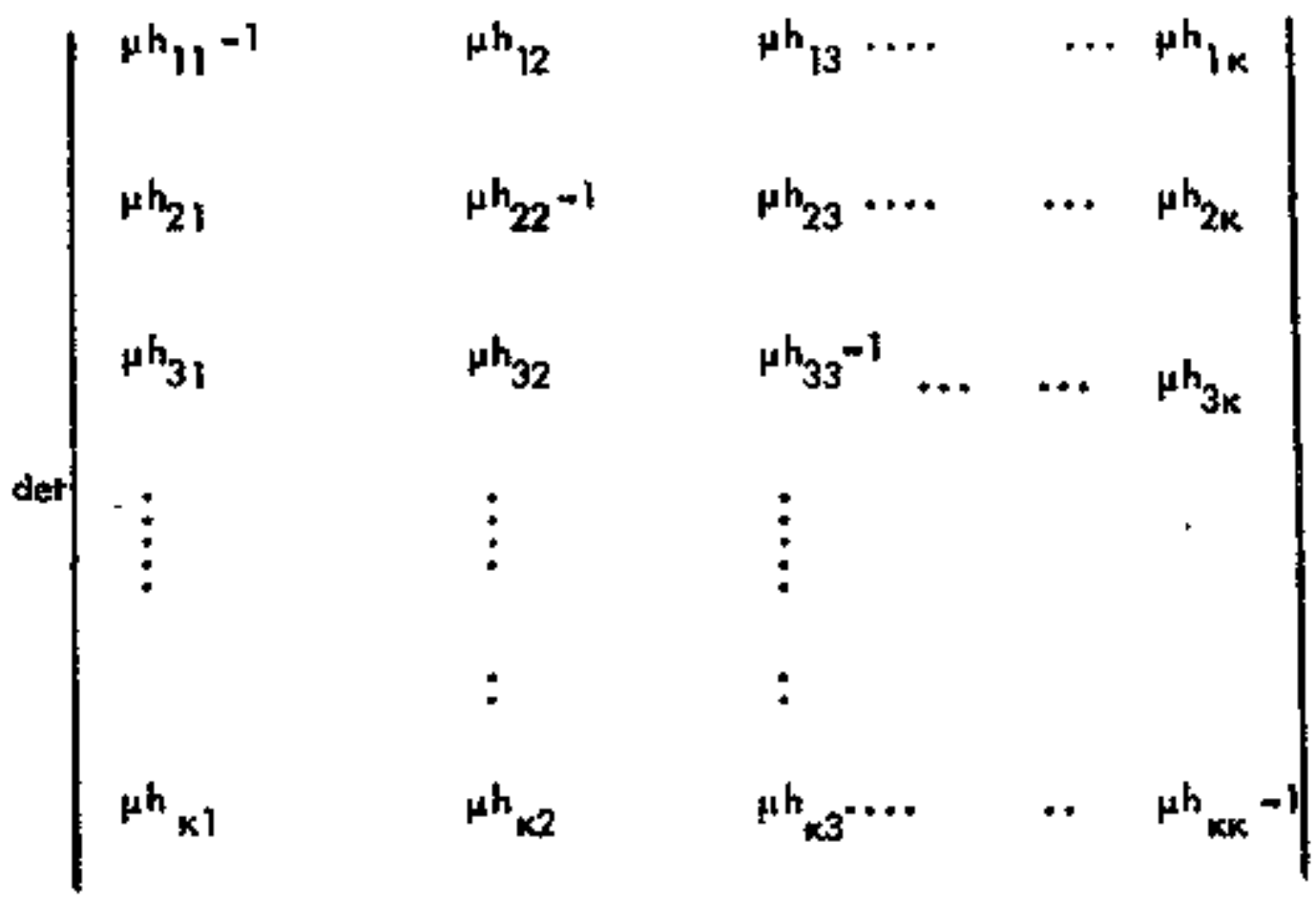

We define on operation $O_{R}$ operoting on any arbitrary matrix $D$ with matrix elements $d_{f j}$ as fallows; $O_{R}$ operating on $D$ has the effect of multiplying the second row by $d_{12}$, the third row by $d_{13}, \ldots . .$. , the $k-t h$ row by $d_{1 k}$ and adding all these to the first now. Thus, under $O_{R}$, the elements of the first now of the transformed matrix $O_{R} D$ becomet

$$
O_{R} D_{1}+d_{11}+\sum_{j=2}^{K} d_{1 j} d_{j i}=d_{1 i}+d_{1 j}^{()} \text {. }
$$

* From now on up to the order desired. 
The remaining rows of $O_{R} D$ are identical to those of $D$. The operation $O_{R}$ has the following useful property

$$
\operatorname{det}\left(O_{R} D\right)=\operatorname{det}(D)
$$

We will exploit this property of $O_{R}$ to compute the secular determinant (5). Let us apply $O_{R}$ to the matrix $\mu H^{(k)}-1$. From (6), we note that the effect of the whole operotion amounts to ereating a polynomial of second order in $\mu$ in the $(1,1)$ - place and tems proportional to $\mu^{2}$ at the $(1, i)-$ places, $1=2,3, \ldots, x$. No other element has changed. If we apply $O_{R}$ successively to the motrix, we keep on inereasing the onder of the polynomial in $\mu$ at the $(1,1)$-place and the power of H at the $(1, i)$-places. Let us apply $O_{R}$ ten times in succession to the matrix. Then, we will hove a polynomial of order eleven in the $(1,1)$-place and terms proportional to $P$ " elsewhere in this row. Only the first row is modified, but other rows remain unehanged. We define onother operotion $O_{C}$, similor to $O_{R}$, except that it operates on the first column and hos the effect:

$$
O_{C} D_{i}^{*} d_{i 1}-d_{i 1}+\sum_{j=2}^{k} d_{i j} d_{j 1}=d_{i 1}+d_{i 1}^{(2)}
$$

Wo operate on the transformed matrix $O_{R}^{10}\left(\mu \mathrm{H}^{(k)}-1\right)$ with $O_{C}$ successivaly nine times to croote terms proportional to $\mu^{10}$ in the $(i, 1)$-place, $i \neq 1$, and o polynomial of order 20 in the $(1,1)$ - ploce. Thus, under the whole oparotion $O_{C}^{9} O_{R}^{10},\left(\mu H^{(k)}-1\right)$ becomess' 


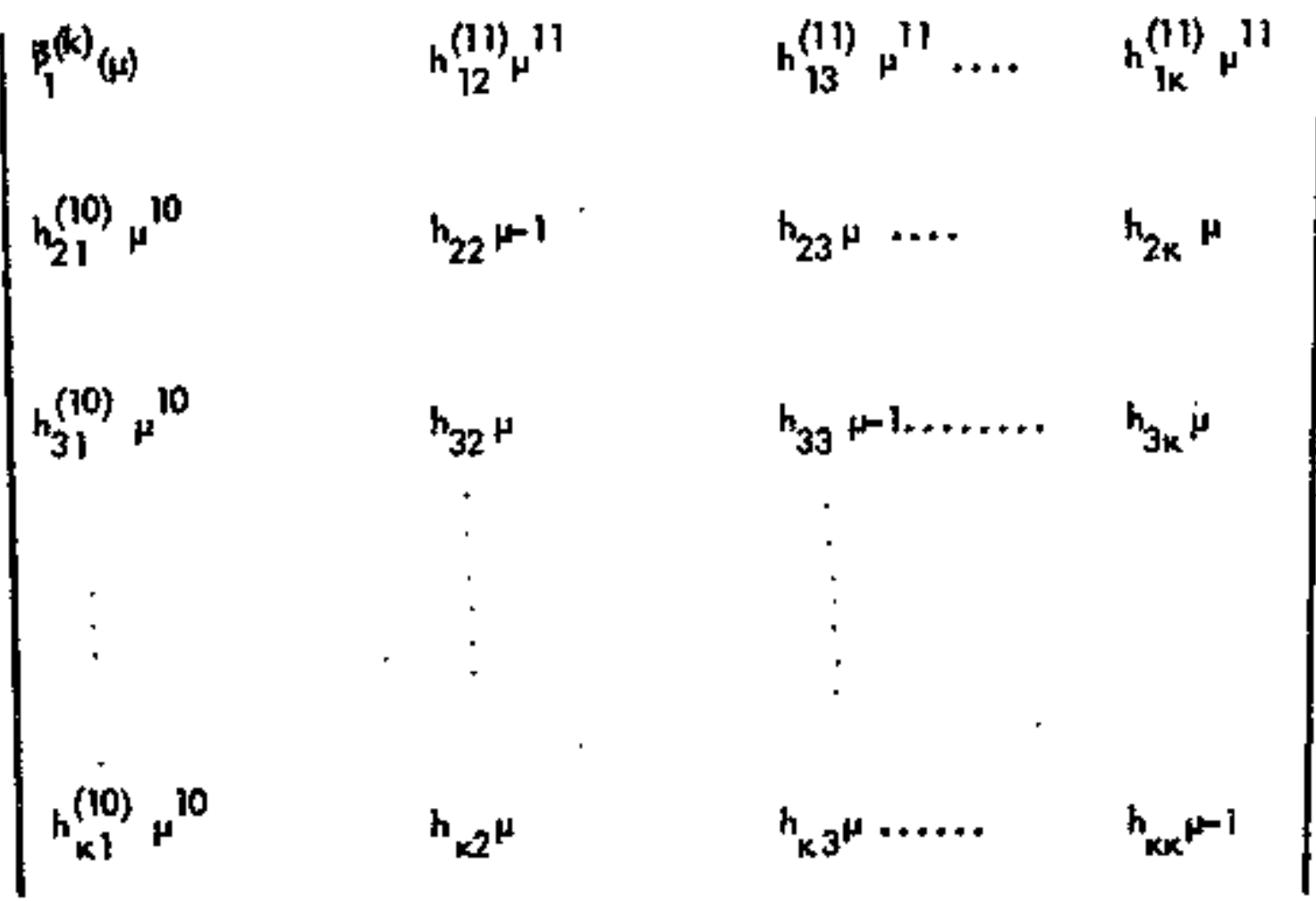

and has the some determinant $\alpha(5)$. Here $P_{1}^{(k)}(\mu)$ is the polynomiol of order 20 mentioned above and involves quantitios $h_{11}^{(m)}, m=1,2 \ldots 20$, defined below. If we now expand the determinant for the matrix (8), we observe that every term in the expension thot does not involve the polynomial $F_{f}^{(k)}(\mu)$ as a factor involves

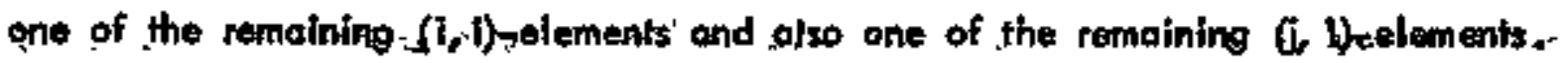

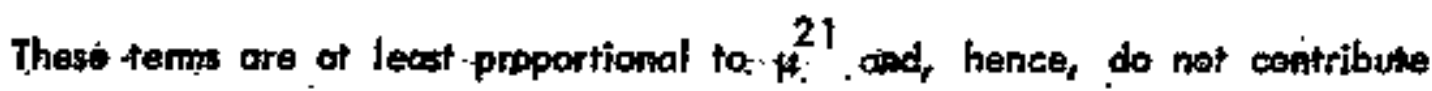
to $\bar{S}^{(k)}(\mu)$. Thus, we can replace each of these elementy by zero and the determinant of this matrix becomes a product of $\beta_{1}^{(k)}(\mu)$ and the determinont of the reduced matrix abtoined by scrotching the first row and first column in (5). We can opply $O_{C}^{9} O_{R}^{10}$ agoin on the reduced matrix and so on, so thot finally, wo have

$$
\beta^{(k)}(\mu)=\beta_{1}^{(k)}(\mu) \beta_{2}^{(k)}(\mu) \ldots \beta_{\vec{k}}^{(k)}(\mu)
$$

where $F_{i}^{(k)}(\mu)$ is the polynomial obtained from the i-th reduced motrix: Of course, 
$-23=$

in the product in (9), we keep toms up to $\mu^{20}$. Itighould also be remarked that

$$
\bar{p}^{(\theta)}(\mu)=\bar{p}^{(n)}(\mu)=\left(Q_{\mu}-1\right)
$$

for the cluster $L_{n, t, T}$.

Let us determine the quantities $\mathrm{u}(\mathrm{m})$ that are required to obtain matrix 8$)$. As $H^{(k)}$ is o symmetric matrix, we find that, if $h_{i j}^{(1)}=h_{i j}$

$$
h_{i j}^{(m)}=i_{i=i+1}^{k} h_{i j^{\prime}}^{(m-1)} h_{i j},
$$

which, for icj, con also be written as

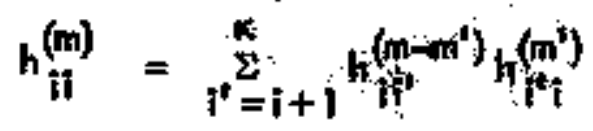

and

$$
h_{i j}^{(m)}=h_{f j}^{(m)}
$$

The polynomial' $p_{i}^{(k)}(\mu)$ involves the quantities $h_{i i}^{(m)}, m=1,2, \ldots .20$. Therefore, we need to compute all $h_{i i}^{(m)}$. We use the first expression of (11) to compute $h_{i j}^{(m)}$ for $m=2,3, \ldots, 10$ and use the second expression to compute $h_{i f}^{(m)}$ for other values of $m$. The polynomial $\tilde{p}_{i}^{(k)}(\mu)$ is given in terms of these quantities as below:

$$
\overrightarrow{\vec{p}}_{f}^{(k)}(\mu)=\sum_{m=1}^{20} h_{j i}^{(m)} \mu^{m}=.
$$


Section 5. Non-Zero Mognetic Field.

We now proceed to discuss the presence of an external magnetic field $\mathcal{S C}$. The Hamfltonian $(1,3)$ is now modified:

$$
H^{\prime}=-J \sum_{(j)} p_{i j}-g \mu_{B} j-s_{3}
$$

where $S_{3}$ is the component of the total spin $\overrightarrow{\$}$ along the direction of the field It; $g$ is the gyromagnetic rotio and $\mu_{B}$ is the Bohr magnetion. As the rwo operatort, $\Sigma P_{i j}$ and $S_{3}$, comanute, wo con write the ron-zero-external-field partition function as

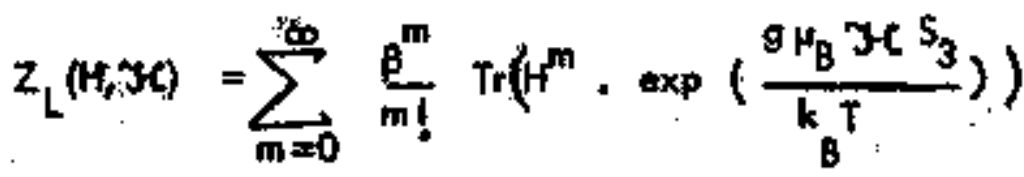

$$
\begin{aligned}
& =\sum_{m=0}^{\infty} \sum_{m !}^{m} \sum_{k=0}^{n} \operatorname{tr}\left\{H^{(k)}\right\}^{m} \exp \quad(n-2 k) \gamma
\end{aligned}
$$

where $\gamma$ is $g \mathrm{~B} Y \mathrm{H} / 2 \mathrm{k}_{B} T$ and we have replaced $S_{3}$ by its value in the invartant subspacei- $R^{(k)}$, given by $(3,3)$. Let us separate out the fertms corresponding to $m=0$ :

$$
z_{L}(H, v c)=\zeta^{n}+\sum_{m=1}^{\infty} \sum_{m_{i}}^{\dot{e}^{m}} \sum_{k=0}^{n} T_{r}\left\{H^{(k)}\right\}^{m}{ }^{n-2 k}
$$

where

$$
\begin{aligned}
& \mu=\theta^{\gamma}: \zeta=\mu+1 / \mu=2 \cosh \gamma \\
& \sum_{k=0}^{n}\left(\begin{array}{l}
n j \\
k
\end{array}\right)^{n-2 k}=\xi^{n}
\end{aligned}
$$


We note thot as $\mathrm{IC} \rightarrow 0, \zeta \rightarrow 2$ for the zero-field cose. We, therefore, define the reduead portition function in anology with that in Section 2 , by dividing by $\xi^{n}:$

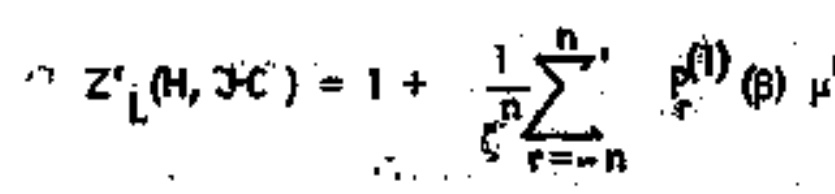

where the polynomial $P_{r}^{(1)}$ (B) is given by

$$
\begin{array}{ll}
P_{r}^{(1)}(p)=\sum_{m=1}^{\infty} \sum_{m i}^{e^{m}} \cdot p_{r, m}^{(1)} & -n \leq r(2) \leq n \\
\therefore & p_{r, m}^{(1)}=\operatorname{Tr}\left\{-H^{\left(\frac{n-r}{2}\right)}\right\}^{m},
\end{array}
$$

The superscript 1 in (6) refers to the fact that the surn over in stats from !

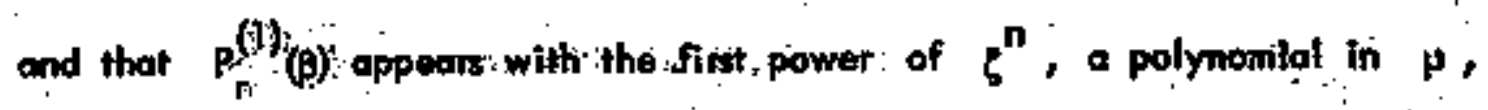
In the denomilinaton the subateript $r$ refers. to the foct that this polynomial is

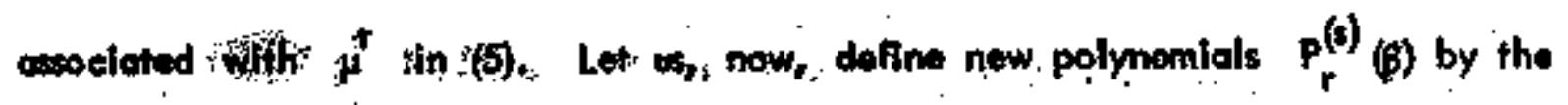
following recursion relation: *

$$
\begin{aligned}
p_{r}^{(s)}(\beta) & =\sum_{r^{\prime}+r^{\prime \prime}=r}^{\prime} p_{r^{(s-1)}}^{(\beta)} p_{t^{\prime \prime}}^{(1)}(\beta) \\
& =\sum_{m=5}^{\infty} \frac{\beta^{(n)}}{m !} \underset{r, m}{p_{r}^{(s)}}
\end{aligned}
$$

* From now on, the symbol $\dot{2}$-will- bo understood as o summotion with an increnent of 2. The range $-r_{0} \leq r(2) \leq r_{0}$. will also be understood in this surse. 
It is acsily seen, from $P_{t, m}^{(1)}=p_{-r, m}^{(1)}$, that $p_{-r}^{(s)}(\beta)=p_{r}^{(s)}(\beta)$. We can now write down an expression for $\ln Z^{\prime},(H, Y C)$ in terms of these polynomials:

$$
\ln Z_{L}^{r}(H, \Im C)=\sum_{m=1}^{\infty} \frac{(-1)^{m+1}}{m} \cdot \frac{1}{\xi^{m n}} \sum_{r=-n m}^{m m} p_{r}^{(m)}(\beta) \mu^{r}
$$

is The above expression $\beta)$ for $\ln Z_{2}^{\prime}(H, J C)$ includes the effect of the external magnetic field $3 \mathrm{C}$ to all ordes in $3 \mathrm{C}$. We observe from $(7)$ that, if we ore interested in the free energy up to ony desired order $\bar{m}$ in $\beta$, wo need to $-\ldots$. compute only the truncated form $\overline{\mathrm{P}}_{\mathrm{r}}^{(s)}(\beta)$ of $(8)$ up to $m=\bar{m}$. This truncated

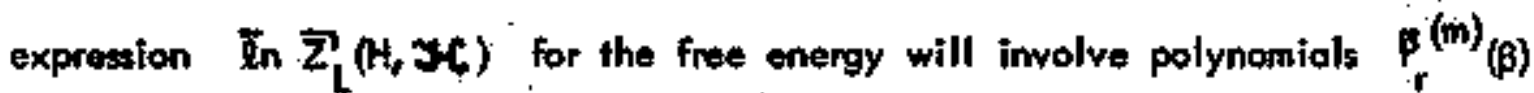
for $m=1,2, \ldots, m$ and $-\mathrm{nm} \leq r(z) \leq n m$. As hos been remarked in the first footnote of Section 2, the finite cluster method works the same woy, oven in the presence of the external field. Therefore, we need to compute the truncated free energies for ofl elusters with $\ell \leq \overline{\mathrm{m}}$. The moximum value of $n$ for these clusters.. wlll be $\vec{m}+1$, corresponding to the Coyley trees. This implies that the highest power of $\zeta$ in the dencininator in the truncated form of $(\beta)$ will be $\bar{m}(m+1)$. Wo will, therefore, rewrite the trinceted form of (8) for every cluster $L_{n, l}, T$ so that every term involves this maximum power of $\zeta$ in the denominator:

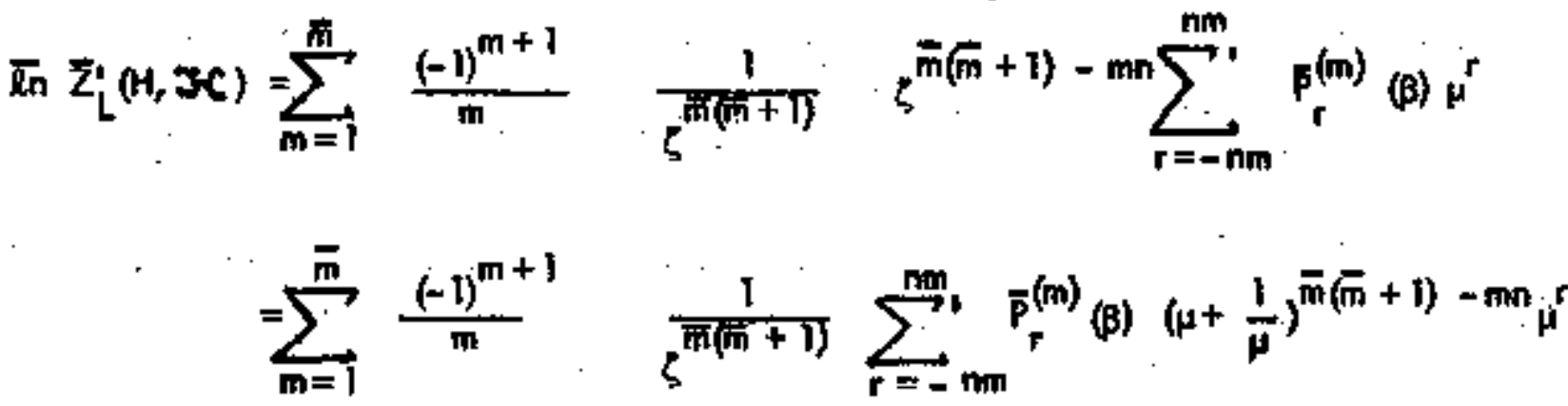

$$
=\frac{1}{\left.\zeta^{m(n+1)}+1\right)} \sum_{r=-\frac{m}{m}(m+1)}^{\left.\sum_{m}^{m}+1\right)} p_{r}(\beta) \mu^{r}
$$


here $\beta,(\beta)$ is the trencated polynomial of the form

$$
P_{r}(\beta)=\sum_{m=1}^{m m} \cdot \frac{\beta^{m}}{m !} P_{s, m} \text {. }
$$

which can be expressed in terms of various $\beta_{r}^{(m)}(\beta)$. The last line in (9) has been abtoined by oollocting all terms for a given $\mu^{r}$.

The above expression (9), however, is not very convenient becauses each power $m$ of $\beta$ involve all $\beta^{r},-\vec{m}(\dot{m}+1) \leq r(R) \leq \bar{m}(\bar{m}+1)$ and will, therefore, involve more and more quantities $P_{p, m}$ for given $m$ as we increase $\bar{m}$. Consider the polynomial $\Sigma^{\prime} P_{r}, m p^{r}$ corrosponding to any given $m$ that oppears in (9). For $m<\bar{m}$, we could heve considered only those finite clusters for which $q \leq m$ and, therefore, the power of $\xi$ in the derfominator in (9) would have been $\xi^{m(m+1)}$ instead of $b^{m(m+1)}$ for such eluaters, Then the polynomial in $\mu$ corresponding to $\beta^{m}$, in $(\theta)$ would heve been $\sum_{r=-m(m+l)}^{m(n+l)} P_{r, m}^{r} p^{r}$, where the $p_{r, m}^{r}$ would be different from the $P_{r_{s}} m^{*}$ it is obvious that

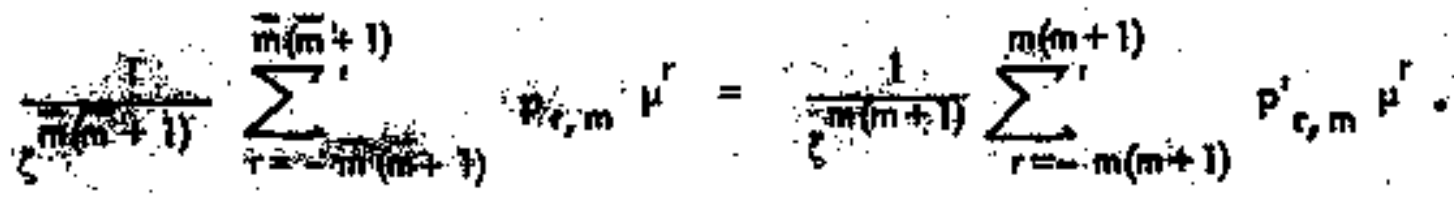

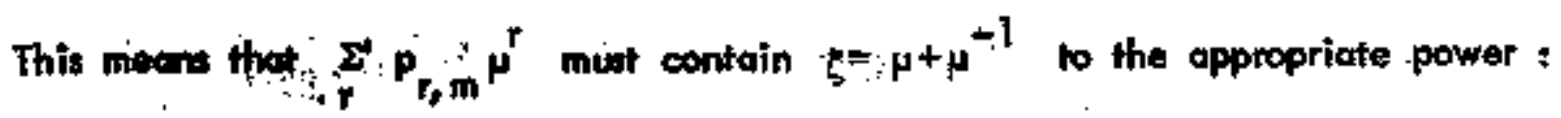

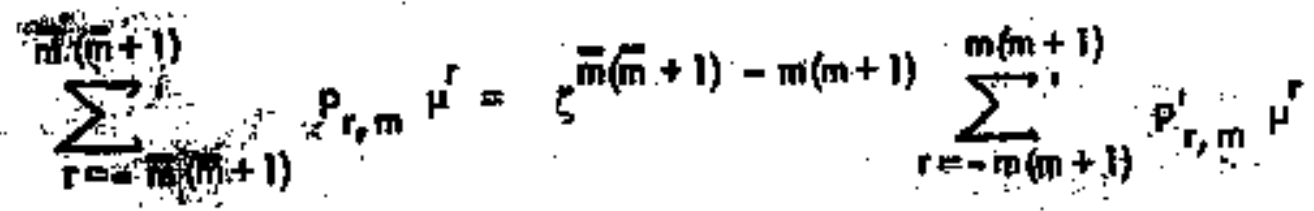

and we can write (9) as

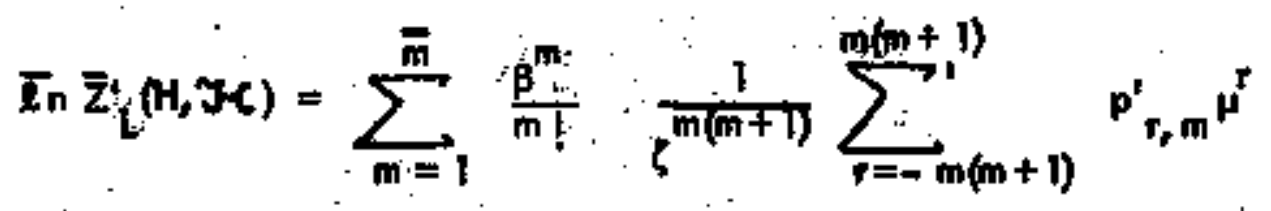


In this expression, thie order of the polynomial in $\mu$ for a given order $m$ in $\beta$ depends only on $m$. The same is frue for the powar of $\zeta$ in the denaminator. Hence, the order of the polynomial in $\mu$ and the power of $\zeta$ in (12) for a glven $\beta^{m}$ do not depend on $n$ and $l$ for any finite eluster $L_{n, k ; t}$ and, therefore, are the same for all finite clusters. 'This is an important obseivation, becouse if implies that in the expression, onologous to (2. 12), in the presence of a mognetic fieldz:

$$
\ln Z_{L}^{\prime}(H, Y C)=\sum_{\Gamma_{n, l, \tau}} \lambda\left(\Gamma_{n, l, \tau}\right) V\left(X-C, \Gamma_{n, l, \tau}\right)
$$

the V-function will be of the form:

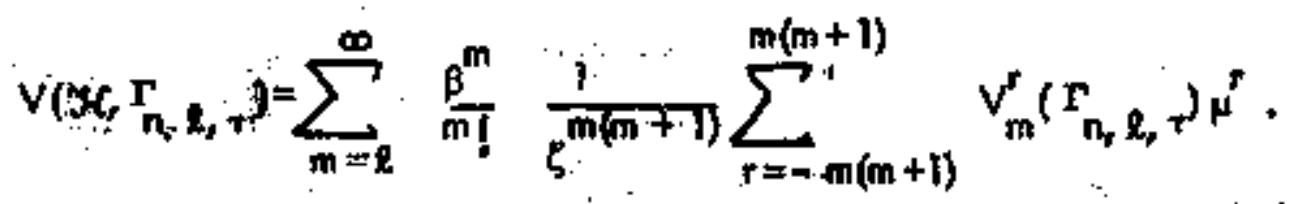

where $V_{m}^{r}$ are integers. The importent foct is that there the order of the

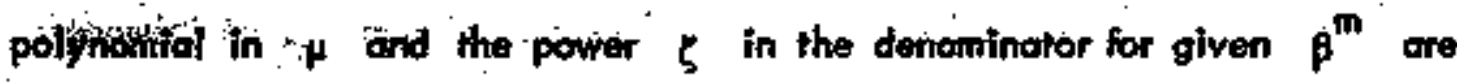

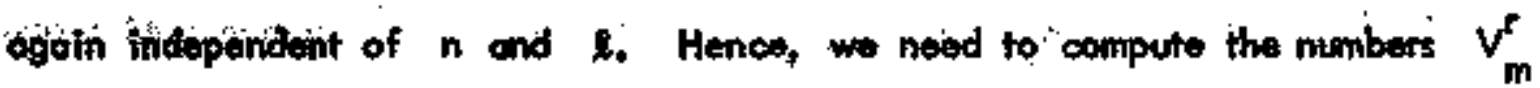
ini (14)' for ooch clustior of Inkerist thid are nectessory for the truicated form

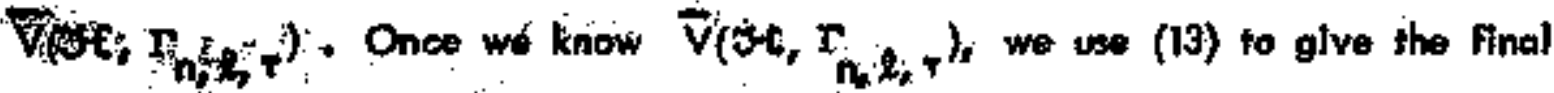

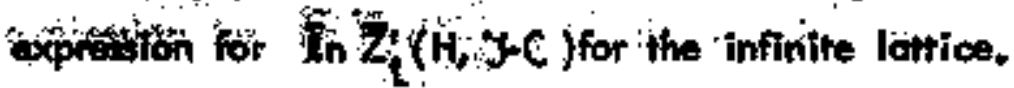

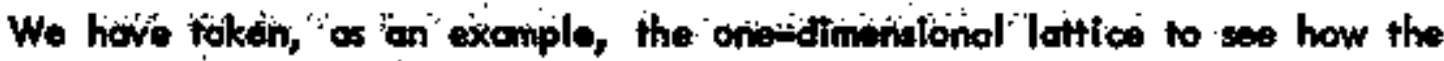
miethod dectiolly works. We compute various quantities : P,r,m and use (11) to deter-

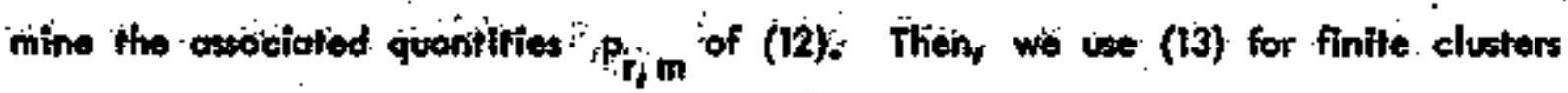

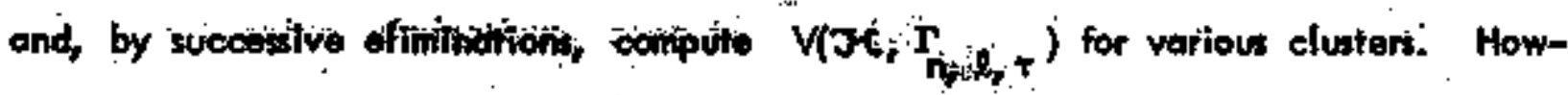

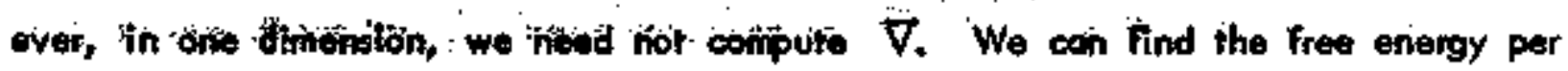


$-29-$

particle in the limit of in infinite lattice as

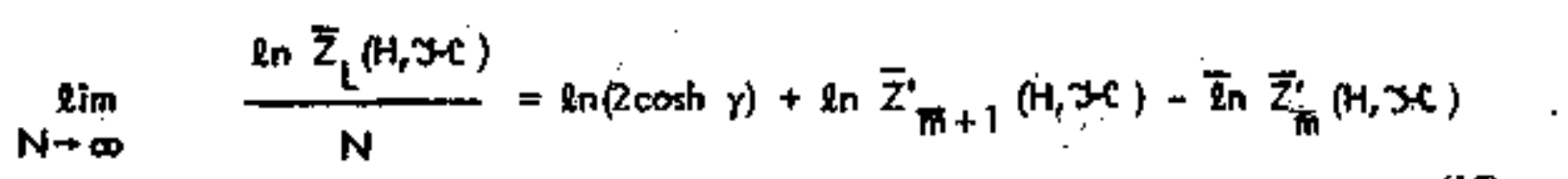

good up to $\bar{m}$-th power in, $\beta$ and all orders in the external magnetic field. The subscripts $\bar{m}+1$ and $\bar{m}$ for $z^{r}$ in (15) refer to the two linear clusters with $\bar{m}+1$ and $\bar{m}$. points respectively. The first term $\ln (Z \cosh Y)$ cones from the term $\zeta^{n}$ required in the definition of the reduced partition function $Z_{L}^{\prime}$.

Woigive the result of our computation in Table 3 , which is good up to fifth order in $\beta$. 
Section 6. Computotion of $V_{m}\left(L_{n, l, r}\right)$ and $\overline{l n} Z_{L}$ :

We will summarize briefly the compurational prodedure to obtein $V_{m}\left(l_{n, l}, \tau\right)$ for various finite elusters which are neoded to find $\ln _{\mathrm{L}} Z_{\mathrm{L}}(\mathrm{H})$ for the infinite regular square lattice. Let us focus our attention on (2. 12) which expresses the relotion between $V_{m}\left(L_{n, l, r}\right)$ and $\ln Z_{L}(H)$. As is clear from (2. 13), each $V_{m}$ appearing in $V\left(L_{n, l, \gamma}\right)$ is non-zero for $m \geq 2$ and appears with $\frac{\beta^{m}}{m !}$ ossociated with it. Thus, if we are interested in computing ln $Z_{L}(1)$ up to twentieth order. in $\beta$, we must include all clusters with $n \leq 20$ and $\ell \leq 20$. However, the presenf method is not suited for clusters of more than ten lattice points ( $>$ > 10), as explained below, and an alternate method is devaloped to include the contribution of graphs thot cover more than ten latsice points. An exception is the elevenmpoint linear eluater which was dealt with with the present method. The altemate method mentioned above wilf be reported in detail in the subsequent publtication where we will give the complate contrtbution to $\ln Z_{L}(H)$ up to mentieth order. The present result for the regular square lattice gives sorrectly the high-femperature expansion of in $Z_{L}$ (H) up to $m=11$ which includes the contributions from all clusters with $n \leq 10$ and $l \leq 10$. The expression for the linear lattice is good up to $m=\mathbf{2 0}$.

Our first problem is to find all possible dusters with $n \leq 10$ on the regular square lattice. We hove drawn all possible topologically distinet clusters explieitly by hand in a systematic woy. We first constructed oll Cayley trees" on the regular square littice up to $n \leq 10$ by systemoticelly odding one point at each step. Then we constructed all stan on the regular lattice with $n \leq 10$. From these two different

* A Cayley tree is a graph which has only one path from ore point to another point. ** A stor is a groph which has no articulation point. 
kinds of clusters, we constructed other possible distinct clusters of mixed kind by rooting Cayley tree elusters on the star elusters or mixed elusters at one or more points. This procedure has enabled us to: construct all possible clusters with $n \leq 10$ on the regular square lattice. It is asy to recognize ropologically equivalent elusters at each stap and, as motter of fact, they are not many. We have con- ' vinced ourselves that we hove not left out any cluster from our tist. We give in Table 1: the number of elusters on a regular square lattice for given pair $(n, l)$; with $n \leq 10$.

The next step is to find the exponsion of the reduced partition function for each of these elusters. We hove written our programming for a general lottice in ony number of dimersions, but af present. We hove restricted aurselves to the regular square latrice. We computed $\mathrm{P}^{(k)}(\mu)$ for $k=1,2, \ldots[n / 2]$ as $\bar{p}^{(0)}(\mu)$ is trivial. We need these truncoted polynomiols in the subspaces $R^{(k)}$ when we want to include the magnetic. field (see Section 5): We will assume HC to be absent in the prosont section. In this case, we do not have to treat various $\beta^{(k)}$ for a cluster seporately and compule $\sum_{i=1}^{20}\left(\lambda_{i}^{(k)}\right)^{m}$ for esch $k$. Insteod; we can multiply them to give the truncated polynomial

$$
\vec{P}(\mu)=\prod_{k=0}^{n} \nabla^{(k)}(\mu)
$$

Now, we compute $p_{m}=i_{i=1}^{2^{n}} \lambda_{i}^{m}$ (see (4.3)). Let $G^{(m)}$ be the set of all free grophs with $m$ bonds defined on $L_{n, l, \tau}$, we thigve:

$$
\sum_{G} T(G)=P_{m} i^{n}
$$

The foctor $2^{\text {n }}$ in (2) is dive to our definition of the reduced portition function

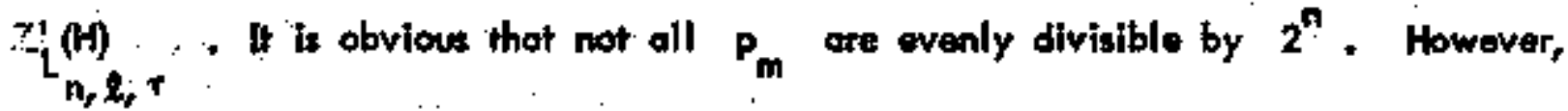


it was observed that for all clusters in Table $1, p_{m}$ ware evenly divisible by $2^{n-4}$ : (See Section 7, where wo discuss this strange property in detail. Now, we can write

$$
\bar{Z}_{L_{n, l_{2}}}(H)=1+\sum_{m=1}^{20} \quad \frac{\beta^{m}}{m_{f}} \frac{\bar{P}_{m}}{2^{4}}
$$

where

$$
\vec{p}_{m}=P_{m} / 2^{n-4}
$$

and

$\bar{l}_{n} Z_{L_{n, \ell, \tau}}(H)=\sum_{m=1}^{20} \frac{(-1)^{m+1}}{m}\left(\Sigma \frac{\beta^{m}}{s !} \frac{\vec{p}_{s}}{2^{4}}\right)^{m}=\underset{m=1}{\sum} \frac{\beta^{m}}{m !} \frac{\bar{q}_{m}}{\left(2^{4}\right)^{m}}$

where $\bar{q}_{m}$ are quantities that are detenminad by various $p_{m^{\prime}}, m^{\prime}=1,2, \ldots m_{0}$. Since $\bar{p}_{m}$ ore integers, $\bar{q}_{m}$ are also integers. Thus, we afways deal with integers throughout our computotion,

The noxt shep in the computation of $V_{m}\left(L_{n, l, T}\right)$ is to determine the cluster constants $\lambda\left(L^{\prime} n^{\prime}, \ell^{\prime}, \tau\right)$ appearing in $(2.15)$ for any finite cluster $L_{n, l, \tau}$. This part is easily programmable. We consider $L_{n, 2}, T$ to be formed by adding one mone bond to $a$ lower clusfer $L_{n-l, l}-1, \tau_{1}$ of a point or to $L_{n, l-1, \tau_{2}}$ between two neighboring points and count all possible subclusters $L_{n^{\prime}, \ell^{\prime}, T^{+}}^{*}$ that include the new bond in $L_{n, \ell_{r}}$. Adding the new subelusters to the list of subelusters for the lower cluster gives us the eluster constants for all subclusters we can embed on the present cluster. ro saye machine time, the machine generated only the list of all possible new subclusters, and the count for each topolegically distinct subetuster wos done by hand. Our results geree with those given by Baker Jnd coworkers $15 \mathrm{~g}$ for clusters with $n \leq 10,2 \leq 10$.

In the case of linear Helsenberg model, we do not need to compute the cluster conshonts, and use $(5.15)$ for $\mathrm{JC}=0$ (cosh $y=1$ ) to give the final expression for the 
free pergy por porticle:

We used the lattice constents for all finite clusters with $n<10, l<10$ given in Ref. $15 g^{*}$ and supplemented these constants for the remaining cluster with \& $>10-n \leq 10$ in order to compute the total contribution of all the finite clusters with $n \leq 10 \cdot \log (\mathrm{n})$

The computation was done on $1 B M-360$ Model 91 of the Columbia University Center for Computing Activitios. The largest postitive integer that can be stored in

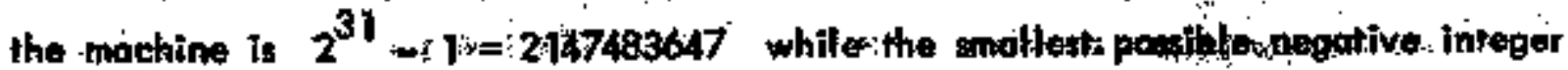
is $-2^{31}$. The quontities $h_{i j}^{(m)}$ of $(4.11)$ and $p_{m}^{(k)}$ are, in foct, much larger integers. For example, one of the typical $\mathrm{A}_{20}^{(5)}$ for o ten-point eluster is

$$
2,6791919667,3248743691,2105621188,8315742179
$$

which, nevertheless, is smaller thon the reol numbers the machine can accopt. However, the rounding error that would be introduced by storing these quantities $h_{1 j}^{(m)}$ and $p_{m}^{(k)}$ os neat numbers-is cumuletive and gives obsuind results. Therefore; it it necory to keep the numbers as integers to ovoid any rounding error. To accomplish thìs, these quantities ore expressed in the base of $2^{31}$, so that any number $\left(n_{k^{\prime}} n_{k-1}, \ldots n_{0}\right)$ with $(k+1)$ ports in this bose has the voluse

$$
n_{k} 2^{31 \cdot k}+n_{k-1} 2^{31(k-1)}+\ldots+n_{1} 2^{31}+n_{0}
$$

For example, the above menentlonid $\mathrm{P}_{20}^{(5)}$ in (5) is written in this representation as

$$
(1259,16.10631343,2018252537,380165924 ; 302057379) \text {. }
$$

The representation is also convenient, we have to divide the quantities $p_{m}$ by numbers of the form $2^{n^{\prime \prime}}$.

*We have taken the correct $\lambda$ for the cluster 865 , which is 6856 . See also, the foetrigte on pose 275, Ref, 21 . 
-34-

In order to save machine core, we represent the number as $\left(-n_{k}, n_{k-1}, n_{k-2}, \ldots, n^{\prime} l^{-n}\right)$ with $k>0$. If, however, $n_{0}$ is zero, we we $-2^{3 l}$. in place of $-n$, which is possible as has been mentioned before. For $k=0$, the number is less then $2^{31}-1$ and is kept as it is. The maximum core that is required is $175 \mathrm{~K}$ for a ten-point cluster. Wo will close this section by giving the approximate machine fine required to compute the twenty quantities $\vec{q}_{m}$ for single cluster with $n>7$ a about 10 second for $n=7$; about 25 seconds for $n=8$; about 1 minute for $n=9$; about 2.75 minutes for $n=10$ and about 7.5 minutes for $n=11$. It is obvious that the present method is not practicable beyond $n=10$. 
Section 7 Chockirg Procedures

A computation of this kind is almays very complieated and it is desirable to have adequate checks on the computational procedure to ensure the accuracy of the results. Finst of all, we hove to be sure thes we hove not left out ony elusters up to $n=10$. Luckily, our list agrees with that of Ref. I5g which, however, gives all finite eluaters if io $n=10$ and $l=10$. Thus, there are a fow more clusters which are not listed there, and we hove convinced oursolves that these ore the only additional distinct clusters that are possible on the regular square lattice. Regarding the lattice constants, we have olready mentioned above that we used the results published in Ref. $15 \mathrm{~g}$, which gives these condtants up to $n=10$ and $\ell=10$. We have also calculated the lattice constants-for the. remaining eluters necsescry for aur results.

We hove remorked above (Section 3) that the traces of vorious powers of the Hamitionian are postive. In fact, all $P_{m}$ are found to be positive for all elusters. As remarked in Section 5 , these $P_{m}$ have a remarkable property: for all clusters, it wos observed that $p_{m}$ were evenly divisible by $2^{n^{t}}$, where $n-n^{4} \leq 4$. The significance of this observation is not obvious. However there seams to be o relotionship between $n-n$ ' and the symmetry of the eluster. The difference $n-n^{\prime}$ is maximum for osymmetric clustors ond decreoses as the symmetry of the cluster increases. It is found that $n \rightarrow n^{\prime}$ is never zero for odd $\ell$, which is due to the fact that $\mathrm{U}\left(\mathrm{om}_{-0}\right)=1 / 2$. However, this difference is zero for a number of elusters with even $l$. For the majority of the clusters, $n-n^{\prime}$ is found to be 3 , but is never more then 4. As a matter of fact, this abservation tumed out to be acheck on the corroctness of the cluster description which is the inpest to the mochine. Whenever $n-n^{\prime}$ was found to be greater than 4 , wiscovered that the eluster wos 
not deseribed correctly. It is this puzzling property of the quentities $P_{m}$ that gives us confidance in our computation. It should be noted that $p_{m}^{(k)}$ do not hove this property as tifopporent from $F^{(k)}(p)=2 p-1$. A similor property is obseived in the cose of $x<\phi$, when we compute the partition function up to $\mathrm{yC}^{2}$. The coefficients of the polynomial proportional : to $\mathrm{JC}^{2}$ heve a similer property. Baker and coworkers 15 d have olso observed o similar property, which eppertifies to the numbers $V_{m}$. Thot property implies that $\bar{q}_{m}$ of $(6.4)$ are integers.

The most important check on the accuracy of our computational procedure comes from the inspection of $v_{m}$. If $l=l_{1}+l_{2}$, where $l_{1}$, is the number of pair-interactions on $L_{n, l, T}$ which, when removed, make the cluster disconnectend and $l_{2}$ is the number of the remaining poir-interactions, then $v_{m}$ vanishes if

$$
m<2 t_{1}+l_{2} \text {. }
$$

(See subsequent publication.) We purposely calculated ofl $v_{m}, m=1,2, \ldots, 20$ so that the property (1) could be chacked for ofl clusters. We observed thot all: these $V_{i n}$ are indeed zero. Incidantly, this check olso gives an indirect check an our computotion of the efuster constonks $\lambda\left(L^{\prime} n^{\prime}, \ell^{\prime}, T^{\prime}\right)$. There were a fow coses whero If revealed an error in the computation of the cluster constants. We belteve that this check is very convincing regarding the occuracy. of our computational procedure. 
Section 8 Results and Final Remarks

The results of our computation in the absence of an external magnetic field are tabuloted in Table 2. We list there the coefficients of that oppear in the high-femperature sories exponsions for the free energy per particle in the thermodymanic limit for the infinite lineor lattice and the infinite regular square lattice;

$$
\lim _{N \rightarrow \infty} \frac{\overline{\ln }_{Z_{L}}(H)}{N}=\ln 2+\sum_{m=1}^{20} \frac{1}{m !}\left(\frac{J}{2 k_{B} T}\right)^{m}{ }_{m}
$$

We have used $\left(J / 2 k_{B} T\right)$ as our exponsion poronteter instead of $\left(1 / k_{B} T\right)$ so that e $m$ ara integers.

The corresponding coefficients in for the usuol Heisenberg model,

$$
H_{\text {Hojsimber }}=-2 J \sum_{a(j)} \vec{s}_{i} \cdot \vec{s}_{j}
$$

con be obtained by the following anbetitutions;

$$
e_{1}^{\text {Heisenberg }}=0
$$

$$
e_{m}^{\text {Heisenberg }}=e_{m} \quad m>1
$$

The values of $e_{\text {, }}$ for the two Homiltonions differ because of the presence of the term $1 / 2$ in (1.3). The presence of this term simply adds a constant ferm to the free onergy and is of no thermodynomic consequence.

Let us first consider the linear lattice. We have considered two lineor clusters, $n=11$ and $n=10$ and report the first twenty tems $e_{1}, e_{2} \ldots, \theta_{20}$ of (1). The known series ${ }^{150}$ for the linoer Heisenberg model indudes all these, toma and 2 ! Heisenberg as weil. We agrae up to $m=19$, but there is a discreponcy between the two values for $m=20$. Our values of $\theta$ are absolutely free from rounding error, but we suspect that those of Rof. 150 ore not. The quentities $\left|e_{1}\right|,\left|e_{2}\right|, \ldots||_{19} \mid$ form on increasing 


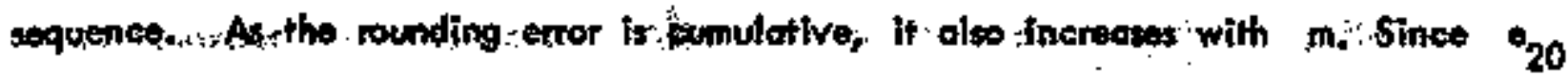

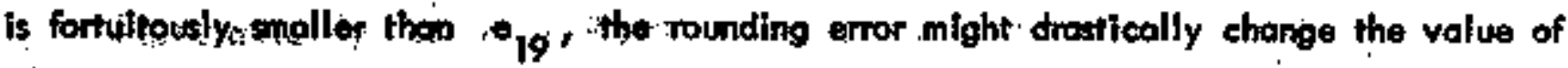
20 . Wo anpect this to bo the cause of the disereponcy.

Let us now consider the regular square lattice for which we heve added all finite clusters with $n \leq 10$ and $\ell \leq 10$ in (2.16). The result it pobulated in Table 2. The known series ${ }^{15 d}$ for the regular square lattice for the usual Helsenberg ipodel gives the first ten quantities $\frac{\text { Heiseriberg }}{m}, m=1,2, \ldots, 70$. The two sets of values for $m=2, \ldots, 10$ agrese completely. This agreement between the two results assures us cormpletely of the accuracy of our computational procedure. As wove not included the contributions of the clusters, with $i>10$, the coefficients ${ }^{\circ} 12^{\prime} \cdots \cdots,{ }^{\circ} 20$ quried in Table 2 are incomplete. But it happens that none of the onitted elusters contributes to ${ }_{11}$. [ In the final version of this paper, we will include the remaining clusters with $n \leq 10$. Besides this, by adding a fow other grophs that contribute to $\mathrm{m}_{\mathrm{m}}$. up to $i n=13$, we will also be able to give the complete volues of $e_{12}$ and $e_{13} . \mathrm{J}$ The rematining coefficients up to $m=20$ will be completed by considering the remaining graph by an alternote method and will be reported in a subsequent publicotion of the series.

In the presence of an externol megrctic fleld NC, we have restricted ourselves to the linear lattice, We hove computed all the terms up to $\beta^{10}$ that are proportional to $y^{2}$ and they ore in complote agreement with the known resulf ${ }^{150}$. We have atso computad the froe eneryy to bil orders in the mognetic field and up to fifth order in $\beta$ to see the fessibility of our mothod as diseussed in Section 5. We tabulote the reault in Table 3 , where we list the coofficionts $\theta_{m, r}, \mathrm{r} \geq 0$ appeoring in

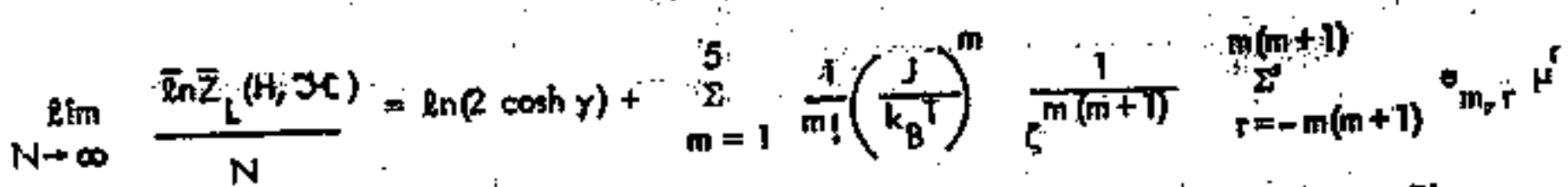




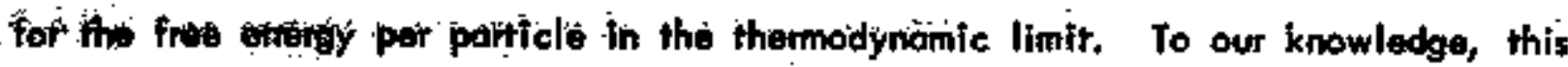
result hises not been givan' before.

Finolly, wo would tike to make the following two remianks, in the first remork we generalize the method discussed in Section 3 to any arbifion spiti. s. The dimention

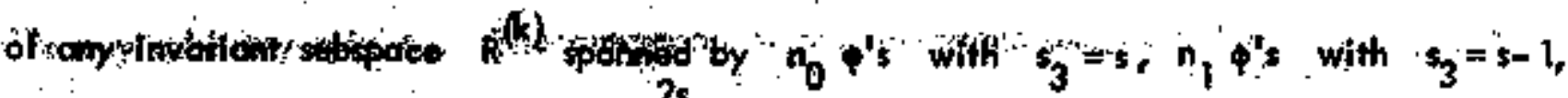

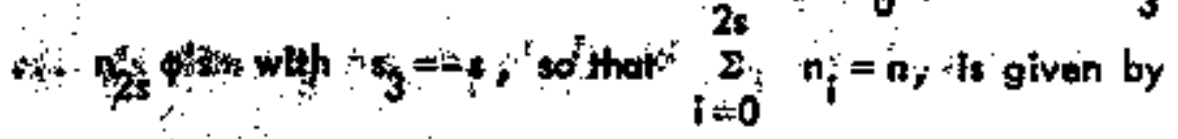
$\therefore$

$$
\frac{n_{i}}{i\left(n_{i} !\right)} \cdot
$$

For $s=1$, the maximum value of (4) for $n=7$ is $7 ! / 3 ! 2 ! 2 !=210^{\circ}$ and for $n=8$ is $8 ! / 3 ! 3 ! 2 !=560$. The moximum dimension increases very quickly with 5 and, therefore, the present mothod comot be carried out beyond certain small values of $n$ in the cose of $s>1 / 2^{i}$ for exomple, the method is nof practicable for $n>7$ in the case $8 \mp 1$ and for $n>6$ in the cose $s=3 / 2$. As the matrix representations for or $H^{(k)}$ do not depend upon sy weed to firid them only for those invoriant

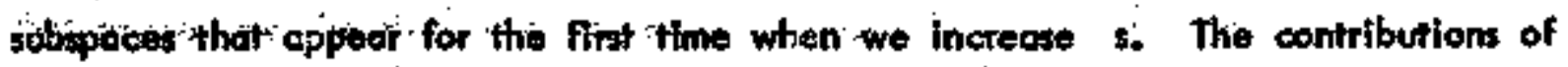
graph covibrifig eluaters with higher $n$ con be calculated by the alternote method to be discussed in $\mathbf{l l}$.

Our serond reinaik concins the chotice of the irivarient subspaces $R^{(k)}$ for

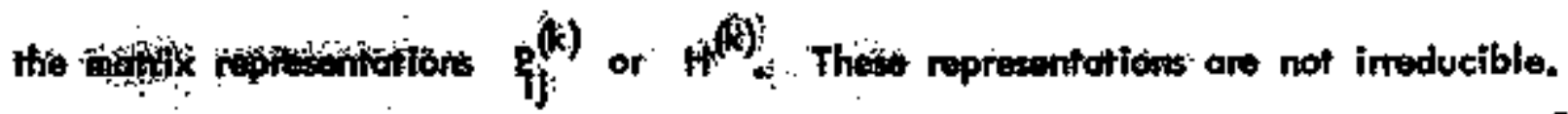

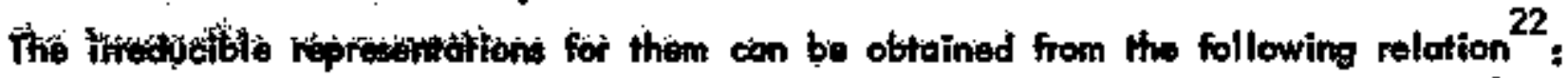

$$
\Delta^{(k)}=0^{(k)} \cdot \Delta^{(k-1)} \quad k \leq[n / 2]
$$

where $\Delta^{(k)}$ represent either $P_{i j}^{(k)}$ or $H^{(k)}$ and $D^{(k)}$ ore the corresponding irreducible 
epresentation, the orden of the matrix representations $D^{(k)}$ are

$$
\left(\begin{array}{c}
n \\
k
\end{array}\right)-\left(\begin{array}{c}
n \\
k-1
\end{array}\right) \text {. }
$$

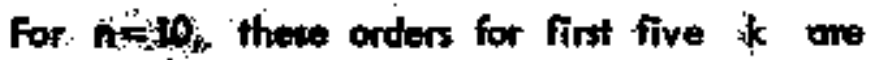

$$
1,9,35,75,90 \text { and } 42 \text {. }
$$

and are considerably amalier than the orders for $\Delta^{(k)}($ see $(3.5))$. Baker and coworkers 15 have uned $\mathrm{D}^{(\mathrm{k})}$ in their work. We, on the other hand, decided to use $\Delta^{(k)}$ primarily because $\Delta^{(k)}$ are parse whereas most of the blements of $D^{(k)}$ are non-zero.

I wish to express my gratitude to Profissor Richord $M$, filedberg for his constant advice and muxt, encosuragement during the progitoss of this work, and for his holpful comments on the monuscript. 
-4 I-

References

1. W. Hoitler and F. London, Z. Physik 44, 455(1927).

2. W. Heisenberg, Z. Physik 49, 619(1928).

3a. P. A.M. Diroc, Proc. Roy.Soc. (London) Al23, 714(1929)

3b. P. A.M. Dirac, The Principles of Quontum Mechonics, Fourth Edition,

(Oxford Univ. Pross, London ond New York 1958).

4. C. Herring, Magnetism: A Treatise on Modern Theory and Motorials,

VoI. IIB, G.T. Rado and H. Suht, eds. (Academic Press, New York and London 1966. .)

5. P.W. Anderson, Solid State Physics: Advances in Research and Applicotions,

Vol, $14, F$. Seltz and D. Tumbulf, eds. (Acodemic Press, New York and London 1963.)

6. K. W. H. Stevens, Mognetiem: A Treatise on Modem Theory and Materials, Vol.1., G.T. Rado and H. Suhi, eds. (Acadomic Press, New York and London 1963.)

7o. W. Opecthowski, Physica 4, 181 (i937).

ד. W. Opechowskt, Physica 6, 1112 (1939).

8., V. Zehler, Z. Naturf. 5A, 344 (1950).

90. G.S. Rushbrooke, and P.J. Wood, Proc. Phys. Soc. (London) A66, $1161(1955)$.

9b. G, S. Rushbrooke and P.J. Wood, Mol.Phys. 1, 257 (1958)

9c. G.S. Rushbrooke and P.J. Wood, Mol. Phys. 6, 409 (1963). 
10. C. Dogb and M.F. Sykes, Proc. Phys, Soc. (London) B69, 486 (1956)

10b. C. Domb and M.F. Sykes, Proce, Phys, Soc, (London) B70, 896 (1957).

11. C. Domb ond D.W. Wood, Phyt, Lett.8, 20 (1964)

Ilbs. C. Domb, N.W. Dalton, G.S. Joyce and D.W. Wood, Proceedings of the International Conference on Mognelism, Nottingham, P.85(1964)

12. D, W. Wood, Phys. Lett. 14, 191 (1965)

13. C. Domb and D.W. Wood, Proc. Phys. Soc. (London) 86, 1 (1965)

14. See Rof. 10a.

C. Domb, Advances in Physics, 35, 245 (1960).

150. G. A. Boker, H., G.5. Rushbrooke and H.E. Gilbert, Phys, Rev, 135A, 1272 (1964) :

15b. G. A. Baker, Jr., H.E. Gilbert, J. Eve and G.S. Rushbrooke, Phys, Lew. 20, $146 \cdot(9966)$.

15c. G.A. Boker, Jr., H.E. Gilbert, J. Eve and G.S. Rushbrooke, Phys. Lett. 22, 269 (1966)

15d. G. A. Baker, Jr., H.E. Gilbert, J. Eve and G.S. Rushbrooke, Phys, Letr. $25 \mathrm{~A}, 207(1967)$.

15. G.A. Baker, tr. and D.S. Gount, Phys, Rev, 155, 545 (1967)

15f. G.A, Baker, Jr., H.E. Gilbert, J. Eve and G.S. Rushbrooke, Phys.'Rev. 164, $800(1967)$

159. G.A. Baker, t5., H.E. Gilbert, J. Evo and G.S, Rushbrooke, Brookhoven Notional Labroatory Repont No. BNL 50053 (1967).

16. C. Dimb, Phil.Mag. Suppl.9, Nos. 34, 35 (1960) .

17. See also Ref. 19.

B. Strieb, H. B. Callen and G. Horowitz, Phys, Rev, 130, 1798 (1963). 
$-43-$

18. GoS. Rushbrooke, J. Math. Phys. 5, 1106 (1964).

19. M. F. Sykes, J.W. Escom, B.R. Heap and B.J. Hilay, L.Moth. Phys. 7,

$$
1557 \text { (1966) . }
$$

20. D. Josnow and M. Wortis, J.Moth. Phiss. 8, 507, (1967)

21. G.S. Rushbrooke, G. A. Baker, Jr. and P. J. Wood, Phiase Tronsitions and

Critical Phonomene, Vol.3, C.Domb and M.S. Greon, eck., (Academic Pross, London and Now York 1974).

22. E.P. Wigner, Group Theory and if Applicotion to the Quontum Mectientes

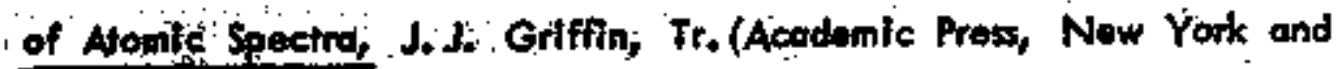
Landon 1959). 


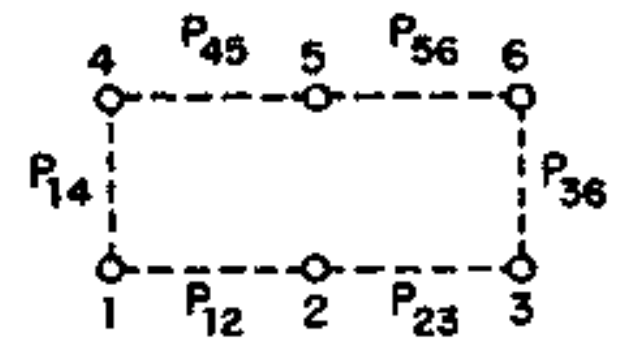

FIG.1 6-POINT, 6-INTERACTION CLUSTER

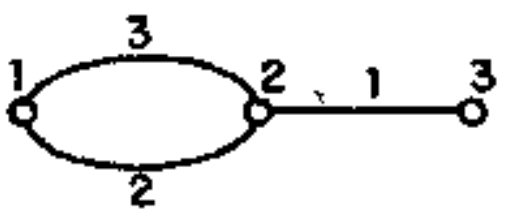

$P_{12} P_{12} P_{23}$

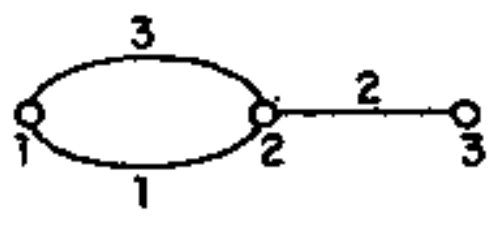

$P_{12} P_{23} P_{12}$

,

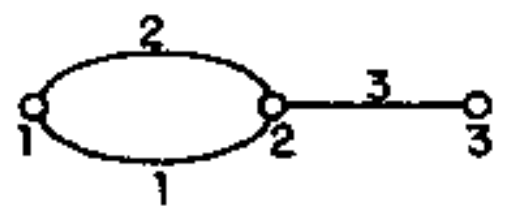

$$
P_{23} P_{12} P_{12}
$$

FIG. 2 LOCALIZED LABELLED GRAPHS

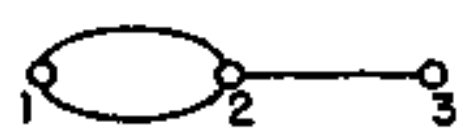

(a)

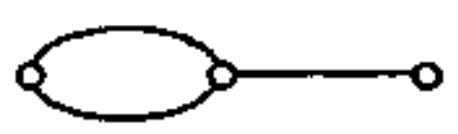

(b)

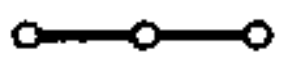

(c)

FIG. 3 (a) LOCALIZED UNLABELLED GRAPH

(b) FREE GRAPH

(c) SKELETON GRAPH 
i
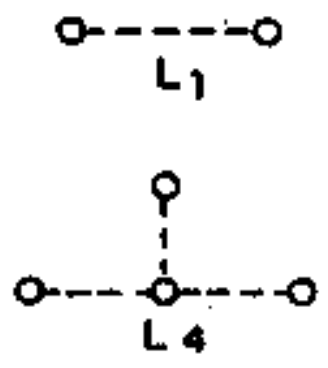
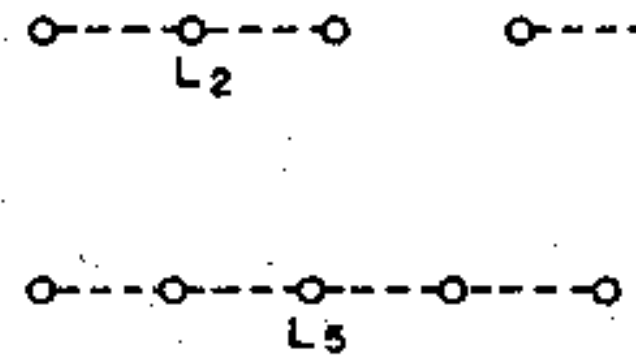

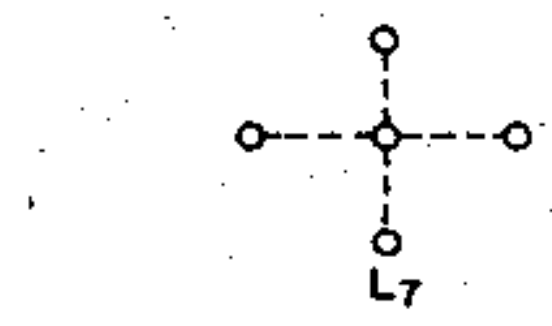

$0-\infty-\infty-\infty$

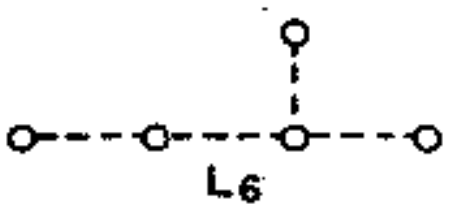

FIG. 4 FIRST SEVEN CLUSTERS 
Toble 1. The number of elusters $L_{n_{t} \ell_{r} T}$ on a regular square lattice for given $n$ and $l$.

\begin{tabular}{|c|c|c|c|c|c|c|c|c|c|}
\hline i & 2 & 3 & 4: & 5 & 6 & 7 & 8 & 9 & 10 \\
\hline$n=1$ & 1 & 1 & 2 & 3 & 5 & 9 & 18 & 35 & 75 \\
\hline$n$ & & & 1 & 1 & 5 & 9 & 30 & 68 & 195 \\
\hline$n+1$ & & & $\cdot$ & & 1 & 3 & 13 & 14 & 128 \\
\hline$n+2$ & & & & & & & 2 & 8 & 43 \\
\hline$n+3$ & & & & & & & & 1 & 4 \\
\hline
\end{tabular}


Table 2. Zero-field Energiess the Coefficients en $_{m} \cdot, \ldots, \ldots, c$

\begin{tabular}{l|r|r|rr|}
\hline & & & & \\
\hline
\end{tabular}

$\because$ * the coefficients ${ }_{12}, \cdots 0_{20}$ are incomplete for the regütar square lattice. twhis number is zero for the uswal Heisenberg model $H=-2 \mathrm{~J} \sum \overrightarrow{\mathrm{s}}^{*} \overrightarrow{\mathrm{F}}^{*}$ j 
rable 3, Non-Zoro-Magnetiofidofd Free Energy for the Lined Lottice; the Coefficients e $m, r$. $+z$

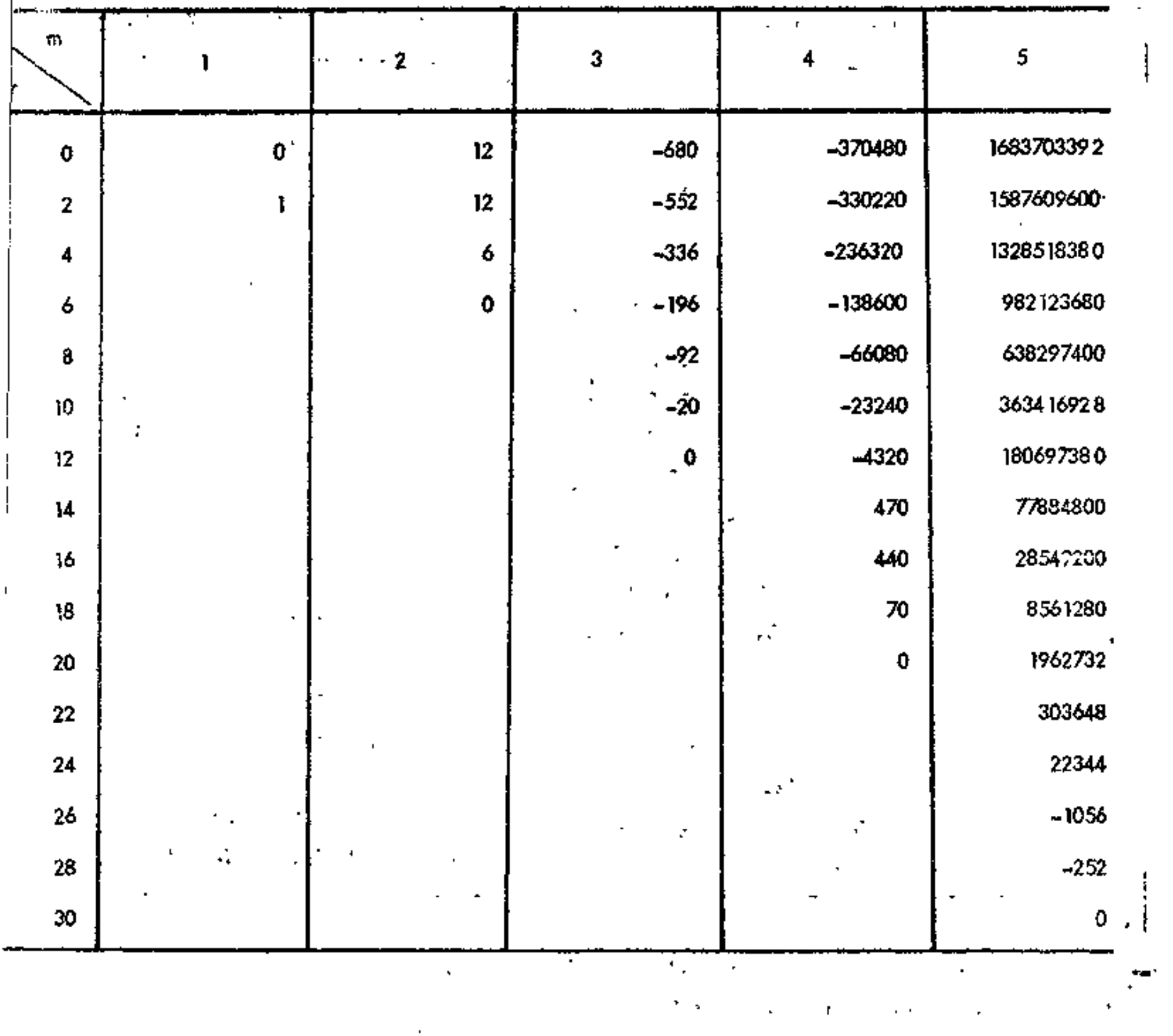

